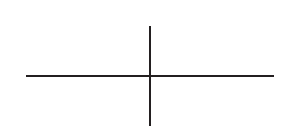

Tohoku Math. J.

57 (2005), 171-200

\title{
TOTAL CURVATURE OF COMPLETE SUBMANIFOLDS OF EUCLIDEAN SPACE
}

\author{
FRANKi DiLlen AND WOLFGANG KÜHNEL
}

(Received June 24, 2003, revised July 21, 2004)

\begin{abstract}
The classical Cohn-Vossen inequality states that for any complete 2-dimensional Riemannian manifold the difference between the Euler characteristic and the normalized total Gaussian curvature is always nonnegative. For complete open surfaces in Euclidean 3space this curvature defect can be interpreted in terms of the length of the curve "at infinity". The goal of this paper is to investigate higher dimensional analogues for open submanifolds of Euclidean space with cone-like ends. This is based on the extrinsic Gauss-Bonnet formula for compact submanifolds with boundary and its extension "to infinity". It turns out that the curvature defect can be positive, zero, or negative, depending on the shape of the ends "at infinity". We give an explicit example of a 4-dimensional hypersurface in Euclidean 5-space where the curvature defect is negative, so that the direct analogue of the Cohn-Vossen inequality does not hold. Furthermore we study the variational problem for the total curvature of hypersurfaces where the ends are not fixed. It turns out that for open hypersurfaces with cone-like ends the total curvature is stationary if and only if each end has vanishing Gauss-Kronecker curvature in the sphere "at infinity". For this case of stationary total curvature we prove a result on the quantization of the total curvature.
\end{abstract}

1. Introduction and main results. The total curvature of Riemannian manifolds and submanifolds has been a field of active research during the last 175 years, initiated by the work of Gauss. For compact manifolds the Gauss-Bonnet theorem is a milestone in differential geometry, in both an extrinsic and an intrinsic version. It states that a certain curvature quantity of the interior of a compact manifold plus another curvature quantity of the boundary (including a discussion of angles if there are any) equals the Euler characteristic, up to a constant depending only on the dimension. The intrinsic version for even-dimensional manifolds is nowadays often called the Gauss-Bonnet-Chern theorem, after Chern [15], [16]. The extrinsic version is closely related with the Hopf index theorem, with the mapping degree of the Gauss map and with the study of critical points of height functions.

In the non-compact case Cohn-Vossen [18] investigated the total curvature of complete open 2-manifolds. In this case the boundary term is missing, and therefore in general the same equality between the total curvature and the Euler characteristic cannot hold. However, the Cohn-Vossen inequality states that the missing boundary term is always nonnegative. Some details on this Gauss-Bonnet difference term will be reviewed in Section 2.

For higher-dimensional open manifolds this missing boundary term is still much less understood, neither extrinsically nor intrinsically. In any case one has to assume that the

2000 Mathematics Subject Classification. Primary 53C40; Secondary 53A07.

This work was done while the second author was a guest of the Katholieke Universiteit Leuven, supported by a fellowship of the research council. 


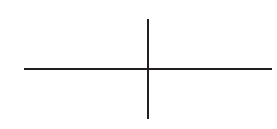

manifold is of finite topology and that the curvature is globally absolutely integrable. Partial results have been obtained by Portnoy [47], Walter [65], Rosenberg [50], Wintgen [70] under certain additional assumptions. For the case of locally symmetric spaces a formula of GaussBonnet type was established, see [44].

For complete manifolds of bounded sectional curvature and finite volume the intrinsic Gauss-Bonnet-Chern theorem still holds, by the work of Cheeger and Gromov [13]. However, this assumption appears to be fairly restrictive in the case of hypersurfaces, because complete hypersurfaces with cylindrical ends have infinite volume, and complete hypersurfaces with finite volume tend to have unbounded sectional curvature at the ends, unless the ends are somehow intrinsically flat, like a cusp over a torus.

If the given manifold is diffeomorphic to the interior of a compact manifold with boundary, then the difference between the Euler characteristic and the total curvature can be expected to depend only on geometric quantities which are defined on this boundary. Intrinsically, the ideal boundary in the sense of Gromov is a concept for defining such a boundary and for studying its geometric properties, see [6]. However, this concept seems to be successful mainly in the case of manifolds of nonpositive sectional curvature. Again, this would exclude the case of hypersurfaces of Euclidean space. For 2-dimensional surfaces, the ideal boundary is in fact a very successful concept in connection with the total curvature, see the discussion in Section 2.

For hypersurfaces or submanifolds of Euclidean $(n+1)$-space $\boldsymbol{E}^{n+1}$ an extrinsic analogue was investigated by Wintgen [70] by means of the set of limit directions. By definition this set is the part of the unit $n$-sphere $S^{n} \subset \boldsymbol{E}^{n+1}$ which appears as the geometric compactification of $M$ "at infinity". If the submanifold behaves "asymptotically cone-like" at the ends (in a sense to be specified below), then the ordinary Gauss-Bonnet theorem implies the following result:

MAIN THEOREM 1. If $M^{n} \subset \boldsymbol{E}^{m+1}$ is a complete $n$-dimensional submanifold with finitely many cone-like ends in Euclidean $(m+1)$-space, then the difference between the Euler characteristic and the total curvature can be explicitly expressed as a sum of the even higher total mean curvatures of the set $M_{\infty} \subset S^{m}$ "at infinity", weighted with certain positive constants:

$$
c_{m} \chi(M)-\int_{\perp^{1}} K d V_{\text {can }}=\sum_{0 \leq 2 i \leq n-1} \frac{c_{m}}{c_{m-n+2 i} c_{n-1-2 i}} \int_{M_{\infty}} K_{2 i} d V_{\infty}
$$

where $c_{j}$ is the volume of the unit standard $j$-sphere.

For more details see Section 5 below. This expression allows a further discussion of the validity of the Cohn-Vossen inequality. It turns out that there is a simple 4-dimensional example in Euclidean 5-space where this inequality does not hold. Remarkably enough, for this example the total curvature is stationary within the class of all submanifolds with cone-like ends. In more generality the variation of the total curvature functional leads to the following: 


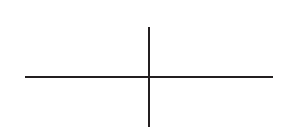

TOTAL CURVATURE OF COMPLETE SUBMANIFOLDS OF EUCLIDEAN SPACE

MAIN THEOREM 2. Let $M^{n} \subset \boldsymbol{E}^{n+1}$ be a complete open hypersurface with finitely many cone-like ends in Euclidean $(n+1)$-space with $n$ even. Then the gradient of the total curvature functional is the Gauss-Kronecker curvature of the hypersurface "at infinity".

For a proof see Theorem 6.7 and Corollary 6.9. Consequently, a formula of GaussBonnet type (i.e., constancy of the total curvature) holds infinitesimally if the Gauss-Kronecker curvature of the hypersurface "at infinity" vanishes identically. In particular, this is the case if the set "at infinity" is totally geodesic. This raises the question for a classification of compact hypersurfaces in the standard unit sphere with vanishing Gauss-Kronecker curvature. One can also ask for the possible values of the total curvature in the stationary case. There is a partial result as follows:

MAIN THEOREM 3. Let $M^{4} \subset \boldsymbol{E}^{5}$ be a complete open hypersurface with finitely many cone-like ends and with stationary total curvature. Assume that for each end the rank of the shape operator in the sphere "at infinity" is constant. Then the normalized total curvature takes values in the integers:

$$
\frac{3}{4 \pi^{2}} \int_{M} K d V \in Z
$$

For a proof, see Theorem 7.6. Note that the analogous result holds for 2-dimensional surfaces with stationary total curvature. Here we have $(1 / 2 \pi) \int_{M} K d A \in \boldsymbol{Z}$. Even though the total curvature functional can attain an interval of values, just by deforming the set at infinity (e.g., ranging from one point to a great sphere), in the stationary case (at least in dimension 2 and 4 ) the total curvature functional ranges only in a discrete set of values, a kind of a quantization of the total curvature.

CONJECTURE (Quantization of the total curvature). For any complete open hypersurface $M^{n} \subset \boldsymbol{E}^{n+1}$ ( $n$ even) with cone-like ends and with stationary total curvature $\int_{M} K d V$, the normalized total curvature $\left(2 / c_{n}\right) \int_{M} K d V$ is an integer.

A short announcement of the main results without proofs appeared in [19].

2. The Cohn-Vossen inequality, intrinsic and extrinsic. For a compact oriented (and connected) Riemannian 2-manifold $(M, g)$ with boundary $\partial M$, the classical GaussBonnet theorem states the equation

$$
2 \pi \chi(M)-\int_{M} K d A=\int_{\partial M} \kappa(s) d s,
$$

where $\kappa$ denotes the geodesic curvature on the oriented boundary. In particular, if all boundary curves are geodesics, we obtain

$$
2 \pi \chi(M)-\int_{M} K d A=0,
$$

the same formula which holds for compact 2-manifolds without boundary.

In the case of non-compact 2-manifolds things are a little bit more complicated. First of all, one should assume that $(M, g)$ is complete because for non-complete metrics one cannot 


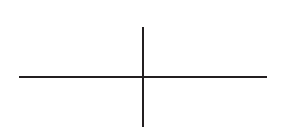

expect general results on the total curvature. Secondly, the quantities $\chi(M)$ and $\int_{M} K d A$ need not be finite numbers. If we assume that $M$ is of finite topological type, then $M$ is homeomorphic to a closed surface $\tilde{M}$ with a finite number of points $p_{1}, \ldots, p_{k}$ removed (called ends), $k \geq 1$. So, in particular,

$$
\chi(M)=\chi(\tilde{M})-k \leq \chi(\tilde{M})-1 \leq 1 .
$$

Finally, one has to assume that the Gauss curvature $K$ is absolutely integrable over $M$, that is, $\int_{M}|K| d A<\infty$. Then the following holds:

THEOREM 2.1 (Cohn-Vossen [18], Satz 6). If $(M, g)$ is a complete Riemannian 2manifold of finite topological type and with absolutely integrable Gauss curvature $K$, then the inequality

$$
2 \pi \chi(M)-\int_{M} K d A \geq 0
$$

holds. In particular, we have $\int_{M} K d A \leq 2 \pi$ if $M$ is non-compact.

There are more subtle versions for the case that $M$ is not of finite topological type (in this case we can formally define $\chi(M)=-\infty)$ and that $\int_{M} K d A$ attains a value in the extended real numbers $[-\infty,+\infty]$. Here the statement is that the Cohn-Vossen inequality still holds. In particular, $\chi(M)=-\infty$ implies $\int_{M} K d A=-\infty$, see [34] and [8]. Under the additional assumption $K>0$ the inequality implies that $\chi(M)>0$, and hence $M$ is homeomorphic to $\boldsymbol{R}^{2}$ unless it is compact. This was independently observed by Cohn-Vossen [18] and by Stoker [61] for surfaces in Euclidean 3-space.

Furthermore, there are a number of additional conditions under which the Gauss-Bonnet equality $2 \pi \chi(M)-\int_{M} K d A=0$ continues to hold in the non-compact case.

THEOREM 2.2. Under the general assumptions of Theorem 2.1 above, any of the following conditions implies the Gauss-Bonnet equality:

(1) (Cohn-Vossen [18, Satz 7]) (M,g) has no end of the type "proper chalice" (german: “eigentlicher Kelch”).

(2) (Huber [34, Thm. 12]) $(M, g)$ is of finite total area $\int_{M} d A$.

(3) (Huber [34, Thm. 11]) For every end there is a sequence of closed curves around it converging to the end such that the length of the curves is uniformly bounded.

(4) (Wintgen [70]) The metric $g$ is induced from a proper immersion $f: M \rightarrow \boldsymbol{E}^{3}$ with finitely many limit directions. The set of limit directions is defined as the set of all possible limits

$$
\lim _{n \rightarrow \infty} \frac{f\left(x_{n}\right)}{\left\|f\left(x_{n}\right)\right\|},
$$

where $x_{n}$ is any sequence in $M$ converging to one particular end.

From the Gauss-Bonnet formula it seems to be obvious that the curvature defect $2 \pi \chi(M)-$ $\int_{M} K d A$ can be calculated or at least controlled by the geodesic curvature of the boundary curves in an exhaustion

$$
M_{1} \subset M_{2} \subset M_{3} \subset \cdots \subset M
$$




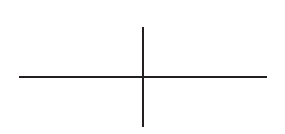

TOTAL CURVATURE OF COMPLETE SUBMANIFOLDS OF EUCLIDEAN SPACE

of the given surface $M$ by compact surfaces $M_{i}$ with boundary. However, it took a surprisingly long time until this curvature defect was well understood.

If a neighborhood of each end is isometric to an open part of a cone with line element $d s^{2}=d r^{2}+c r \cdot d s_{1}^{2}$ (where $c$ is a constant and $d s_{1}^{2}$ is the line element of the unit circle), then the contribution of such an end to the curvature defect is nothing but $2 \pi c$, in particular, it is $2 \pi$ if the end is planar. By passing to the limit, the same holds if the metric is asymptotically conelike at each end or, in the extrinsic setting, if the surface is asymptotically cone-like itself. More specific results in that direction were obtained for minimal surfaces in $\boldsymbol{E}^{3}$ because the end of each minimal surface is asymptotically planar. For embedded and complete minimal surfaces with finite total curvature Osserman [45] showed the equation

$$
2 \pi \chi(M)-\int_{M} K d A=2 \pi k,
$$

where $k$ is the number of ends, see also [38] for the case of immersed minimal surfaces and "multiplicities" at the ends. This result was generalized by White [68] to the case of complete surfaces in $\boldsymbol{E}^{n}$ such that the norm of the second fundamental form is square integrable. Note that this norm square equals $\kappa_{1}^{2}+\kappa_{2}^{2}=-2 \kappa_{1} \kappa_{2}=-2 K$ for minimal surfaces.

For an arbitrary complete surface in $\boldsymbol{E}^{3}$ the asymptotic behavior of the metric near the ends was used by Rosenberg [49] for obtaining a short proof of the Cohn-Vossen inequality. The curvature defect was further studied by Shiohama. He obtained the following result:

THEOREM 2.3 ([56]). Let $(M, g)$ be a complete 2-dimensional Riemannian manifold of finite topology and finite total curvature. Then

$$
2 \pi \chi(M)-\int_{M} K d A=\lim _{t \rightarrow \infty} \frac{L(t)}{t}=\lim _{t \rightarrow \infty} \frac{2 A(t)}{t^{2}},
$$

where $L(t)$ denotes the length of the geodesic distance circle in distance $t$ and $A(t)$ denotes the area of the geodesic disc with radius t. The center point of the disc is arbitrary.

For special cases of Shiohama's result compare the preceding papers [30, 26, 24]. Again the Cohn-Vossen inequality follows from Shiohama's theorem because length and area are nonnegative quantities.

Wintgen [70] suggested that the curvature defect of a complete and properly immersed surface in Euclidean 3-space is the length of the set $M_{\infty}$ of the so-called limit directions $\lim _{n \rightarrow \infty} f\left(x_{n}\right) /\left\|f\left(x_{n}\right)\right\|$. He conjectured that one can always assign a finite length to this set if the total curvature is finite. Unfortunately, this is not true in general, not even if the norm of the second fundamental form is square integrable, a stronger assumption. White [68] gave the following example: Take the surface which over the $(x, y)$-plane is parameterized as the graph of the function $z=x \sin \left(\log \log \left(1+\sqrt{x^{2}+y^{2}}\right)\right)$. Here the set of limit directions covers an open part of the unit 2-sphere.

Intrinsically, one can relate the curvature defect with the so-called ideal boundary in the sense of [6]. The following theorem was obtained by Shioya [58, 59], compare [42, 72]: 


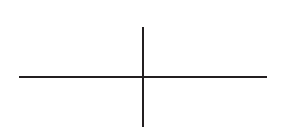

THEOREM 2.4. For the curvature defect of a complete Riemannian 2-manifold $M$ with finitely many ends $E_{1}, \ldots, E_{k}$ and finite total curvature the following formula holds:

$$
2 \pi \chi(M)-\int_{M} K d A=\sum_{i=1}^{k} l_{i},
$$

where $l_{i}$ denotes the length of the ideal boundary associated to the end $E_{i}$.

Again the Cohn-Vossen inequality is a corollary because a length is always nonnegative. Yim [72] interpreted this nonnegativity in terms of the convexity of the ideal boundary of the surface at infinity. More information on Section 2 can be found in [73].

3. The extrinsic Gauss-Bonnet theorem. For investigating higher dimensional analogues of the classical Gauss-Bonnet formula for 2-manifolds, one can first look at the integrated extrinsic curvature of a compact hypersurface. Here an appropriate curvature is the Gauss-Kronecker curvature $K=K_{n}$ which is defined as the determinant of the shape operator, where $n$ is the dimension of the manifold. In the even-dimensional case this curvature is independent of the unit normal, while in the odd-dimensional case its sign depends on the unit normal. It is well-known that $K$ is intrinsic if $n$ is even. For the history of the following theorem compare [29].

Notation. The constant $c_{n}$ denotes the volume of the standard unit $n$-sphere. This can be expressed in terms of the Gamma function as follows: $c_{n-1}=2 \pi^{n / 2} / \Gamma(n / 2)$. The symbol $d V$ denotes the volume element of a submanifold, sometimes in the form $d V_{M}$ for specifying the manifold on which it is defined.

THEOREM 3.1 (Gauss-Bonnet-Hopf [31], [32], Satz VI). Let $M^{n} \subset \boldsymbol{E}^{n+1}$ be an embedded compact hypersurface such that $M$ is the boundary of its interior $M_{\mathrm{int}} \subset \boldsymbol{E}^{n+1}$, and let $K$ denote the Gauss-Kronecker curvature of $M$ with respect to the inner normal (pointing to $\left.M_{\mathrm{int}}\right)$. Then the following hold:

(i) $\int_{M} K d V_{M}=c_{n} \cdot \chi\left(M_{\text {int }}\right)$.

(ii) If $n$ is even, then $\chi(M)=2 \chi\left(M_{\mathrm{int}}\right)$ and, consequently, $\int_{M} K d V_{M}=\left(c_{n} / 2\right)$. $\chi(M)$. Moreover, this equality holds for arbitrary immersions $f: M \rightarrow \boldsymbol{E}^{n+1}$ of a compact orientable $n$-manifold, even if $M$ is not the boundary of any $(n+1)$-manifold.

Hopf called the type of hypersurfaces in (i) Jordan hypersurfaces. The essential difference between even and odd dimensions is that for odd dimensions the total curvature is not a topological invariant of the hypersurface, whereas for even dimensions the Gauss-Bonnet equation (ii) holds independently of the nature (or even the existence) of an interior $M_{\text {int }}$. In the non-orientable case in (ii) one can pass to the 2-sheeted orientable covering. (i) can be extended to the case of immersions $f: M_{\text {int }} \rightarrow \boldsymbol{E}^{n+1}$ if $M_{\text {int }}$ is a given $(n+1)$-manifold with boundary. As a matter of fact (already mentioned in [32]), for odd dimensions the total curvature does depend on the choice of $M_{\text {int }}$, i.e., on the choice of the embedding. 


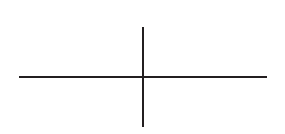

TOTAL CURVATURE OF COMPLETE SUBMANIFOLDS OF EUCLIDEAN SPACE

EXAMPLE 3.2. Let $S^{1} \subset \boldsymbol{E}^{2} \subset \boldsymbol{E}^{4}$ be the standard unit circle and $\left(S^{1}\right)_{\varepsilon}$ its $\varepsilon$-tube in Euclidean 4-space. Similarly, let $S^{2} \subset \boldsymbol{E}^{3} \subset \boldsymbol{E}^{4}$ be the standard unit sphere and $\left(S^{2}\right)_{\varepsilon}$ its $\varepsilon$ tube in Euclidean 4-space. Then $\left(S^{1}\right)_{\varepsilon}$ and $\left(S^{2}\right)_{\varepsilon}$ are both diffeomorphic to $S^{1} \times S^{2}$. However, the total Gauss-Kronecker curvature is zero in the first case and positive in the second case, according to $\chi\left(\left(S^{1}\right)_{\leq \varepsilon}\right)=\chi\left(S^{1}\right)=0$ and $\chi\left(\left(S^{2}\right)_{\leq \varepsilon}\right)=\chi\left(S^{2}\right)=2$.

Nevertheless, we have the following folklore result:

Proposition 3.3. Within the class of all immersions $f: M^{n} \rightarrow \boldsymbol{E}^{n+1}$ of a fixed compact manifold $M$, the total Gauss-Kronecker curvature $\int_{M} K d V_{M}$ depends only on the regular homotopy class of $f$.

This follows from the variational formula for the total curvature, see Section 5. The gradient of the curvature functional $\int_{M} K d V$ is identically zero. Of course, Proposition 3.3 is interesting only for odd $n$. In this case it reduces the problem of determining all possible values for the total curvature to determining all regular homotopy classes of immersions together with examples in each class for which the total curvature can be calculated. See [74] for interesting results. In the case of planar curves Proposition 3.3 is well-known by the WhitneyGraustein theorem because $(1 / 2 \pi) \int_{c} \kappa d s$ of a closed curve $c$ equals the rotation index of $c$. The theorem on turning tangents (the "Hopf Umlaufsatz") can be regarded as the special case $n=1$ in Theorem 3.1 (i).

In the case of submanifolds of higher codimensions one has to regard the so-called Lipschitz-Killing curvature, which is defined as the determinant of the shape operator $A \xi$ in direction of a specific unit normal $\xi$ (for the background compare [40])

$$
\left\langle A_{\xi}(X), Y\right\rangle=\left\langle\nabla_{X} Y, \xi\right\rangle .
$$

Therefore integrating the curvature requires the space of all unit normals at all points

$$
\perp^{1}(M)=\left\{(p, \xi) \mid p \in M,\|\xi\|=1, \xi \perp T_{p} M\right\}
$$

which is nothing but the total space of the unit normal bundle of an embedding or immersion. For a submanifold $M^{n} \subset \boldsymbol{E}^{m+1}, \perp^{1}(M)$ can be regarded as a submanifold of the tangent bundle of $\boldsymbol{E}^{m+1}$, or as a submanifold of $M \times \boldsymbol{E}^{m+1}$. This space $\perp^{1}(M)$ carries a canonical orientation (induced by the outer normal) which is compatible with the orientation of the ambient space, and it carries a so-called canonical volume form $d V_{\text {can }}$ which is induced from this orientation, see [21]. Locally we have $d V_{\text {can }}=d V_{M} \wedge d V_{S^{m-n}}$. Note that the manifold $\perp^{1}$ is orientable even for immersions of non-orientable manifolds. An orientation of $\perp^{1}$ is obtained by the choice of either of the following:

$$
\begin{array}{cl}
(p, \xi) \mapsto \xi \quad \text { (the outer normal) } \\
(p, \xi) \mapsto-\xi \quad \text { (the inner normal) } .
\end{array}
$$

REMARK 3.4. In the sequel, $K$ or $K_{n}$ denotes the Lipschitz-Killing curvature, where $n$ indicates the dimension of the manifold on which it is defined. More precisely, we use the symbol $K(\xi)$ or $K_{n}(\xi)$ for the Lipschitz-Killing curvature in the direction of a unit normal $\xi$. 


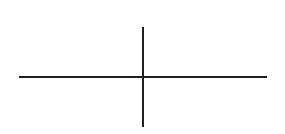

THEOREM 3.5 ([1,20]). Let $M^{n} \subset \boldsymbol{E}^{m+1}$ be an embedded compact submanifold without boundary (or an immersion of $M$ ), and let $K$ denote the Lipschitz-Killing curvature, defined on the unit normal bundle $\perp^{1}(M)$. Then the Gauss-Bonnet formula holds in the following form:

$$
\int_{\perp^{1}(M)} K d V_{\text {can }}=c_{m} \cdot \chi(M) .
$$

Moreover, if $m$ is even, we have $\chi\left(\perp^{1}(M)\right)=2 \chi(M)$.

Note that by a linear standard embedding $\boldsymbol{E}^{m+1} \rightarrow \boldsymbol{E}^{m+2}$ one can always make the dimension of the ambient space an even number. If $n$ is odd, then by the obvious equation $K(-\xi)=(-1)^{n} K(\xi)$ the total Lipschitz-Killing curvature is pointwise zero, and hence the equation above becomes trivial. On the other hand, it leads to a geometric interpretation for the equation $\chi(M)=0$ for odd-dimensional manifolds $M$, if one uses the fact that every manifold can be embedded somehow into some Euclidean space.

A "modern" proof reduces Theorem 3.5 to the Hopf index theorem for nondegenerate height functions, see [21, p. 28]. Nevertheless, it is kind of interesting that independently Allendoerfer and Fenchel proved this at about the same time and by essentially the same method, namely, by the method of tubes, thus by reducing it to the Gauss-Bonnet-Hopf theorem. We briefly sketch this proof, for later use in Theorem 3.7.

PROOF. For an embedding or immersion $f: M^{n} \rightarrow \boldsymbol{E}^{m+1}$ of a compact manifold $M$ and for sufficiently small $\varepsilon>0$ the $\varepsilon$-tube defines an embedding or immersion $f_{\varepsilon}: \perp^{1} \rightarrow$ $\boldsymbol{E}^{m+1}$ by

$$
f_{\varepsilon}(p, \xi)=f(p)+\varepsilon \xi
$$

If $M^{n} \subset \boldsymbol{E}^{m+1}$ is an embedded or immersed manifold, then the Gauss-Bonnet-Hopf theorem 3.1 for the tube $M_{\varepsilon}$ (or $f_{\varepsilon}$ ) and for the inner unit normal states that

$$
\int_{\perp^{1}(M)} K_{m} d V_{\varepsilon}=c_{m} \cdot \chi(M),
$$

because $M_{\varepsilon}$ is the boundary of the solid (embedded of immersed) $\varepsilon$-tube $M_{\leq \varepsilon}$ which has the same Euler characteristic as $M$ itself. Here we use the fact that the Gauss-Bonnet-Hopf theorem remains valid for immersions of the manifold $N$ bounding the given $M$. Furthermore, from the additivity of the Euler characteristic we obtain the relation

$$
\chi\left(\perp^{1}\right)=\chi\left(S^{m-n}\right) \cdot \chi(M),
$$

which for even $m$ leads to

$$
\chi\left(\perp^{1}\right)=2 \chi(M)
$$

because $\chi(M)=0$ if $n$ is odd. Therefore the proof of the Allendoerfer-Fenchel theorem is completed by the equation

$$
\int_{\perp^{1}} K_{m} d V_{\varepsilon}=\int_{\perp^{1}} K_{n} d V_{\text {can }},
$$

which holds for embeddings and immersions and for an arbitrary dimension and codimension by the following pointwise observation. 


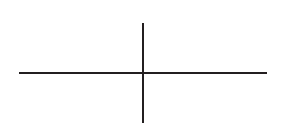

TOTAL CURVATURE OF COMPLETE SUBMANIFOLDS OF EUCLIDEAN SPACE

If $\kappa_{1}(\xi), \ldots, \kappa_{n}(\xi)$ denote the principal curvatures of $M$ at $p$ in direction $\xi$, then the principal curvatures of $M_{\varepsilon}$ at $(p, \xi)$ in direction $-\xi$ are

$$
\frac{-\kappa_{1}(\xi)}{1-\varepsilon \kappa_{1}(\xi)}, \ldots, \frac{-\kappa_{n}(\xi)}{1-\varepsilon \kappa_{n}(\xi)}, \underbrace{\frac{1}{\varepsilon}, \ldots, \frac{1}{\varepsilon}}_{m-n} .
$$

The volume forms $d V_{\varepsilon}$ and $d V_{\text {can }}$ on $\perp^{1}$ are related by the equation

$$
d V_{\varepsilon}=\prod_{i=1}^{n}\left(1-\varepsilon \kappa_{i}(\xi)\right) \varepsilon^{m-n} d V_{\mathrm{can}},
$$

and hence $K_{m} d V_{\varepsilon}=(-1)^{n} K_{n} d V_{\text {can }}$. However, for odd $n$, the integral $\int_{\perp^{1}} K_{n} d V_{\text {can vanishes }}$ pointwise, and thus we have

$$
\int_{\perp^{1}} K_{m} d V_{\varepsilon}=\int_{\perp^{1}} K_{n} d V_{\mathrm{can}}
$$

in any case.

In order to extend the extrinsic Gauss-Bonnet theorem to compact submanifolds with boundary, one has to find an appropriate analogue for the right hand side boundary term in the classical formula

$$
2 \pi \chi(M)-\int_{M} K d A=\int_{\partial M} \kappa(s) d s .
$$

In any case we have to distinguish between inner points $p \in M \backslash \partial M$ and boundary points $p \in \partial M$. In the interior the curvature will be defined as above, i.e., at a point $p \in M \backslash \partial M$ we consider the curvature

$$
K(p)=\int_{\xi \in \perp_{p}^{1}} K_{n}(\xi) d V_{S^{m-n}}
$$

and the total Lipschitz-Killing curvature

$$
\int_{p \in M \backslash \partial M} K(p) d V_{M}=\int_{\perp^{1}(M \backslash \partial M)} K_{n} d V_{\text {can }} .
$$

At the boundary it is quite natural to consider only the outer unit normals and to integrate only over the set

$$
\perp_{+}^{1}(\partial M)=\left\{(p, \xi) \mid p \in \partial M,\|\xi\|=1, \xi \perp T_{p} \partial M,\left\langle\xi, v_{\text {out }}\right\rangle \geq 0\right\},
$$

where $v_{\text {out }}$ denotes the unique outer unit normal vector, which is tangent to $M$, which is perpendicular to $\partial M$ and which points away from $M$. Hence $(p, \xi) \in \perp_{+}^{1}(\partial M)$ if and only if $\xi$ has a nonpositive inner product with the tangent $\dot{c}(0)$ of any smooth curve $c:[0,1) \rightarrow M$ with $c(0)=p \in \partial M$.

DEFINITION 3.6 (unit normal space, total curvature). For a compact submanifold $M^{n} \subset \boldsymbol{E}^{m+1}$ with boundary $\partial M$ we define the unit normal space $N^{1}$ by

$$
N^{1}=\perp^{1}(M) \cup \perp_{+}^{1}(\partial M) .
$$




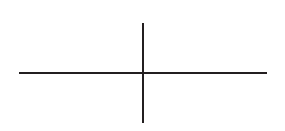

It carries a canonical volume form $d V_{\text {can }}$ as in the case of a submanifold without boundary. Then the total curvature of $M$ is defined as the sum of the total curvatures of the two parts from $\perp^{1}(M \backslash \partial M)$ and from $\perp_{+}^{1}(\partial M)$ :

$$
\mathrm{TC}(M, \partial M):=\int_{N^{1}} K d V_{\mathrm{can}}=\int_{\xi \in \perp^{1}(M \backslash \partial M)} K_{n}(\xi) d V_{\mathrm{can}}+\int_{\xi \in \perp_{+}^{1}(\partial M)} K_{n-1}(-\xi) d V_{\mathrm{can}} .
$$

The signs are chosen in view of the Gauss-Bonnet-Hopf theorem 3.1 for the interior of the tube $M_{\varepsilon}=\left\{p+\varepsilon \xi \mid(p, \xi) \in N^{1}\right\}$, where we have to take the inner normal along the boundary. The analogous definition applies to immersions $f:(M, \partial M) \rightarrow \boldsymbol{E}^{m+1}$ with the tube $f_{\varepsilon}(p, \xi)=f(p)+\varepsilon \xi$.

With this definition the Allendoerfer-Fenchel tube argument can be carried over to the case of compact submanifolds with boundary (and immersions of such) as follows.

THEOREM 3.7. For a compact submanifold $M^{n} \subset \boldsymbol{E}^{m+1}$ with boundary $\partial M$ (or an immersion of $M$ ) the Gauss-Bonnet formula holds as follows:

$$
\mathrm{TC}(M, \partial M)=c_{m} \cdot \chi(M) .
$$

Moreover, if $m$ is even, then we have $\chi\left(N^{1}\right)=2 \chi(M)$.

PROOF. The key observation is the equation

$$
\mathrm{TC}(M, \partial M)=\int_{M_{\varepsilon}} K_{m} d V_{\varepsilon}
$$

for sufficiently small $\varepsilon>0$, where $K_{m}$ is taken with respect to the inner normal of the tube $M_{\varepsilon}$. Then in the second step we obtain

$$
\int_{M_{\varepsilon}} K_{m} d V_{\varepsilon}=c_{m} \cdot \chi\left(M_{\leq \varepsilon}\right)=c_{m} \cdot \chi(M)
$$

from the Gauss-Bonnet-Hopf theorem 3.1. Finally, we need the equation

$$
\chi\left(N^{1}\right)=\left(1+(-1)^{m}\right) \chi(M),
$$

which we obtain as follows: From the additivity of the Euler characteristic we get

$$
\chi\left(N^{1}\right)=\chi(M)\left(1+(-1)^{m-n}\right)-\chi(\partial M)(-1)^{m-n} .
$$

For even $m$ this leads to $\chi\left(N^{1}\right)=2 \chi(M)$.

For the first step $\operatorname{TC}(M, \partial M)=\int_{M_{\varepsilon}} K_{m} d V_{\varepsilon}$, we use the formulae for the tube above.

For any $(p, \xi) \in \perp^{1}(M \backslash \partial M)$ we have the same situation as in the proof of Theorem 3.5 above. If $(p, \xi) \in \perp^{1}(\partial M)$, then we similarly have

$$
K_{m}(-\xi) d V_{\varepsilon}=(-1)^{n-1} K_{n-1}(\xi) d V_{\text {can }}=K_{n-1}(-\xi) d V_{\text {can }} .
$$

Again, for odd $n$, the integral $\int_{\left(\perp^{1}\right)_{p}} K_{n} d V_{S^{m-n}}$ vanishes pointwise at any interior point $p \in$ $M \backslash \partial M$, and we obtain

$$
\int_{M_{\varepsilon}} K_{m} d V_{\varepsilon}=\int_{\xi \in \perp^{1}(M \backslash \partial M)} K_{n}(\xi) d V_{\mathrm{can}}+\int_{\xi \in \perp_{+}^{1}(\partial M)} K_{n-1}(-\xi) d V_{\mathrm{can}}
$$




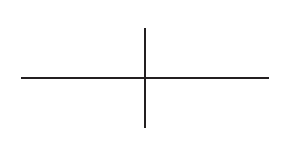

TOTAL CURVATURE OF COMPLETE SUBMANIFOLDS OF EUCLIDEAN SPACE

$$
=\frac{1}{2} \int_{\xi \in \perp_{+}^{1}(\partial M)}\left(K_{n-1}(-\xi)+K_{n-1}(\xi)\right) d V_{\mathrm{can}}=\frac{1}{2} \int_{\xi \in \perp^{1}(\partial M)} K_{n-1} d V_{\mathrm{can}} .
$$

For an intrinsic version of Theorem 3.7 see $[2,53]$.

COROLlary 3.8. (i) If $n$ is odd, then we have $\chi(\partial M)=2 \chi(M)$ and, consequently,

$$
\operatorname{TC}(M, \partial M)=\frac{1}{2} \int_{\perp^{1}(\partial M)} K_{n-1} d V_{\text {can }}=\frac{c_{m}}{2} \chi(\partial M)=c_{m} \chi(M) .
$$

Therefore, in this case Theorem 3.7 is equivalent to the statement of the Gauss-Bonnet theorem 3.5 for the boundary $\partial M$.

(ii) We have

$$
\mathrm{TAC}(M, \partial M)=\operatorname{TAC}(M \backslash \partial M)+\frac{1}{2} \mathrm{TAC}(\partial M),
$$

where TAC denotes the total absolute curvature defined as the integral over the absolute value of the Lipschitz-Killing curvature.

The formula in (ii) is useful for studying tight immersions, i.e., such immersions for which the TAC attains its minimum value, see [7]. The problem of minimum total absolute curvature was investigated [17], compare [11]. Tightness for complete non-compact submanifolds was studied in [28].

By Definition 3.6 and by Theorem 3.7 the Gauss-Bonnet difference term

$$
c_{m} \chi(M)-\int_{\perp^{1}(M \backslash \partial M)} K_{n} d V_{\text {can }}
$$

can be expressed as the integral of $K_{n-1}$ over the set of outer unit normals at $\partial M$. Obviously, any $\tilde{\xi} \in\left(\perp_{+}^{1}\right)_{p}$ can be uniquely written as

$$
\tilde{\xi}=\cos \varphi \cdot v_{\text {out }}+\sin \varphi \cdot \xi,
$$

where $0 \leq \varphi \leq \pi / 2$ and $\xi$ is a unit normal vector to $M$ at $p \in \partial M$. Vice versa, any such $\xi$ leads to a $\tilde{\xi}$ in $\left(\perp_{+}^{1}\right)_{p}$ for any $\varphi$ with $0 \leq \varphi \leq \pi / 2$. This enables us to compute this integral by Fubini's theorem, pointwise evaluated for the normal sphere $S^{m-n}$ on the one hand and half the normal sphere $S^{m-n+1}$ on the other hand.

In view of an exhaustion of a noncompact manifold by compact manifolds with boundary, the Gauss-Bonnet defect $c_{m} \chi(M)-\int_{\perp^{1}(M \backslash \partial M)} K_{n} d V_{\text {can }}$ is closely related to this "outer curvature" of the "ideal boundary" in the sphere at infinity. For this purpose we first formulate the following theorem for submanifolds in the unit ball which can be regarded as a model for the Euclidean space after compactification by a unit sphere at "infinity".

THEOREM 3.9 (Gauss-Bonnet theorem for submanifolds in the closed unit ball). Let $\left(M^{n}, \partial M^{n}\right) \subset\left(B^{m+1}, S^{m}\right)$ be a compact submanifold which is orthogonal at the boundary, i.e., the outer normal $v_{\text {out }}$ of $M$ at each boundary point coincides with the outer normal 


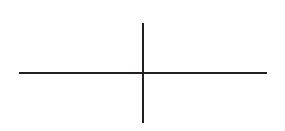

of $S^{m}$. Then for the Gauss-Bonnet defect the equation

$$
c_{m} \chi(M)-\int_{\perp^{1}(M \backslash \partial M)} K d V_{\text {can }}=\sum_{0 \leq 2 i \leq n-1} \frac{c_{m}}{c_{m-n+2 i} c_{n-1-2 i}} \int_{\perp^{1}(\partial M)} K_{2 i} d V_{\text {can }}
$$

holds, where $K_{j}$ denotes the $j^{\text {th }}$ elementary symmetric function of the eigenvalues of the shape operator of the embedding $\partial M \rightarrow S^{m}$.

The right hand side was called the outer curvature in [27]. The total curvatures

$$
\boldsymbol{K}_{j}=\int_{\perp^{1}} K_{j} d V_{\text {can }}
$$

themselves play an important role in integral geometry and differential geometry, see [52]. In particular they are intrinsic invariants if $j$ is even, see [36] and Section 4.

PROOF. At each boundary point $p \in \partial M$ we compute the boundary term as follows:

$$
\begin{aligned}
\int_{\tilde{\xi} \in\left(\perp_{+}^{1}\right)_{p}} K_{n-1}(-\tilde{\xi}) d V_{S^{m-n+1}}=\int_{\xi \in \perp_{p}^{1}, 0 \leq \varphi \leq \pi / 2} K_{n-1}\left(\sin \varphi \cdot \xi-\cos \varphi \cdot v_{\text {out }}\right) d V_{S^{m-n+1}} \\
=\int_{\perp_{p}^{1}} \int_{0}^{\pi / 2} \operatorname{det}\left(\sin \varphi \cdot A_{\xi}-\cos \varphi \cdot A_{v_{\text {out }}}\right) d V_{S^{m-n}} \wedge \sin ^{m-n} \varphi d \varphi \\
=\int_{\perp_{p}^{1}} \int_{0}^{\pi / 2} \sin ^{m-1} \varphi \operatorname{det}\left(A_{\xi}+\cot \varphi \cdot \text { Id }\right) d V_{S^{m-n}} \wedge d \varphi \\
=\int_{\perp_{p}^{1}} \int_{0}^{\pi / 2} \sin ^{m-1} \varphi \sum_{j=0}^{n-1} K_{j}(\xi) \cot ^{n-1-j} \varphi d V_{S^{m-n}} \wedge d \varphi \\
=\sum_{j=0}^{n-1} \int_{0}^{\pi / 2} \sin ^{m-n+j} \varphi \cos ^{n-1-j} \varphi d \varphi \int_{\xi \in \perp_{p}^{1}} K_{j}(\xi) d V_{S^{m-n}}
\end{aligned}
$$

Note that in our case the shape operator $A_{\xi}$ of $\partial M$ in the ambient Euclidean space coincides with the shape operator of $\partial M$ in $S^{m}$ and that $A_{v_{\text {out }}}$ is nothing but the negative identity, namely, the shape operator of $S^{m} \subset \boldsymbol{E}^{m+1}$. The last integral vanishes for odd $j$, and so we obtain the sum over all even $j=2 i$. The proof is completed by the equation

$$
\int_{0}^{\pi / 2} \sin ^{m-n+j} \varphi \cos ^{n-1-j} \varphi d \varphi=\frac{c_{m}}{c_{m-n+j} c_{n-1-j}} .
$$

The key observation for this proof has been used for similar problems, e.g., for the study of tubes, see [67]. For the case $n=m$ the statement of Theorem 3.9 can be found in [27].

COROLlaRY 3.10 (Special cases). (1) For a compact surface $\left(M^{2}, \partial M^{2}\right) \subset\left(B^{3}, S^{2}\right)$ of this type we have

$$
4 \pi \chi(M)-2 \int_{M} K d V_{M}=2 \cdot \operatorname{length}(\partial M)
$$




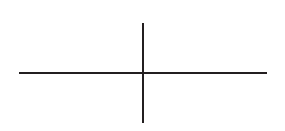

TOTAL CURVATURE OF COMPLETE SUBMANIFOLDS OF EUCLIDEAN SPACE

(2) For a compact hypersurface $\left(M^{4}, \partial M^{4}\right) \subset\left(B^{5}, S^{4}\right)$ of this type we have

$$
\frac{8}{3} \pi^{2} \chi(M)-2 \int_{M} K_{4} d V_{M}=\frac{1}{3} \int_{\partial M}(S-2) d V_{\partial M},
$$

where $S$ denotes the scalar curvature of $\partial M^{4}$.

PROOF. From the formula in Theorem 3.9 we obtain

$$
c_{2} \chi(M)-\mathrm{TC}(M \backslash \partial M)=\frac{c_{2}}{c_{0} c_{1}} \int_{\partial M} 2 K_{0} d V_{\partial M}=2 \int_{\partial M} d V_{\partial M}
$$

in the case (i). For (ii) we have

$$
\begin{gathered}
\frac{8}{3} \pi^{2} \chi(M)-\int_{\perp^{1}(M)} K_{4} d V_{\text {can }}=\frac{8}{3} \pi^{2} \int_{\partial M}\left(\frac{2}{4 \pi^{2}}+\frac{2 K_{2}}{8 \pi^{2}}\right) d V_{\partial M} \\
=\frac{1}{3} \int_{\partial M}\left(4+2 K_{2}\right) d V_{\partial M}=\frac{1}{3} \int_{\partial M}(S-2) d V_{\partial M},
\end{gathered}
$$

where $S=6+2 K_{2}$ is the scalar curvature of $\partial M$.

If $n$ is odd then in Theorem 3.9 the contribution of $M \backslash \partial M$ is zero, and for the boundary we can express the total outer curvature in two different ways. This leads to the following

COROLLARY 3.11 (Integral formulae for total curvatures in $S^{m}$ ). Let $N^{n-1} \subset S^{m}$ be an even-dimensional compact submanifold. Then

$$
\frac{c_{m}}{2} \chi(N)=\sum_{0 \leq 2 i \leq n-1} \frac{c_{m}}{c_{m-n+2 i} c_{n-1-2 i}} \int_{\perp^{1}(N)} K_{2 i} d V_{\text {can }},
$$

where $K_{2 i}$ denotes the $2 i^{\text {th }}$ elementary symmetric function of the eigenvalues of the shape operator of $N \subset S^{m}$.

This equation is often called the Allendoerfer-Weil formula in the sphere, although it is not explicitly given in [2]. It can be found in [36, p. 248], and for hypersurfaces it is stated in $[62$, p. 261] together with a differential topological proof.

In the case where $n=m=3$ the equation in Corollary 3.11 is nothing but the integral of the classical Gauss equation $K=1+K_{2}$, where $K$ denotes the inner Gaussian curvature and $K_{2}$ the extrinsic determinant of the shape operator. Hence the extrinsic "total curvature defect" $2 \pi \chi(N)-\int_{N} K_{2} d V_{N}$ becomes strictly positive.

For $n=m=5$ this equation takes the form

$$
\frac{4}{3} \pi^{2} \chi(N)-\int_{N} K_{4} d V_{N}=\frac{1}{6} \int_{N}(S-6) d V_{N},
$$

where $S$ denotes the scalar curvature of $N^{4}$. Hence the integral mean value 6 for the scalar curvature is the critical value which determines the extrinsic "total curvature defect". Compare the critical value 2 in the 3 -dimensional case in Corollary 3.10.

PROOF OF 3.11. It is sufficient to consider the case that two congruent copies of $N$ are the common boundary of an immersed cylinder $\tilde{N}=N \times[0,1]$ in the ball $B^{m+1}$, which is orthogonal at the two boundaries. Then the total curvature of the interior part vanishes, and 


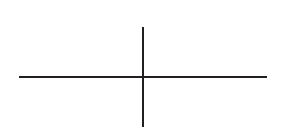

the assertion follows directly from Theorem 3.9, since each of the two boundary components contributes the same value.

4. The pointwise intrinsic nature of the total curvature. It is known that the Lipschitz-Killing curvature of an even-dimensional submanifold is an intrinsic quantity when integrated over the unit normal bundle at a point $p$, see [67], [52]. The same holds for the even total curvatures, see [36], [14]. In this section we give an independent and elementary proof for the fact that the even total curvatures

$$
\int_{\perp_{p}^{1}} K_{2 i}(\xi) d \xi
$$

indeed are intrinsic invariants. In particular, this applies to the Lipschitz-Killing curvature of even-dimensional submanifolds. We give a detailed proof for the latter case which is based on a formula for the Laplacian of the determinant. The general case is proved similarly, we will only sketch it.

4.1. The Lipschitz-Killing curvature. Let $M^{2 n}$ be a submanifold in $\boldsymbol{E}^{m+1}=\boldsymbol{E}^{2 n+k}$ and let $p \in M$. Let $\perp_{p}^{1}$ be the unit sphere in the normal space at $p$. Let $\omega$ be the volume element of $M$ at $p$, and let $\left\{e_{1}, \ldots, e_{2 n}\right\}$ be an orthonormal basis of $T_{p} M$. We define a function

$$
f: \perp_{p}^{1} \rightarrow \boldsymbol{R}: \xi \mapsto \omega\left(A_{\xi} e_{1}, \ldots, A_{\xi} e_{2 n}\right) .
$$

Note that $f(\xi)=\operatorname{det} A_{\xi}$. For $\xi \in \perp_{p}^{1}$, let $\left\{\xi_{1}, \ldots, \xi_{k-1}\right\}$ be an orthonormal basis of $T_{\xi} \perp_{p}^{1}$. Putting $\xi=\xi_{k}$, we obtain an orthonormal basis $\left\{\xi_{1}, \ldots, \xi_{k-1}, \xi_{k}\right\}$ of the normal space at $p$. If $\Delta$ denotes the ordinary Laplacian of $\perp_{p}^{1}$, then we have

$$
\Delta f(\xi)=\left.\sum_{\alpha=1}^{k-1} \frac{d^{2}}{d t^{2}}\right|_{t=0} f\left(\cos t \cdot \xi+\sin t \cdot \xi_{\alpha}\right) .
$$

Then, by a straightforward computation, it follows that

$$
\begin{aligned}
\Delta f(\xi)= & \sum_{\alpha=1}^{k-1}\left((-2 n) f(\xi)+\sum_{i \neq j} \omega\left(A_{\xi} e_{1}, \ldots, A_{\xi_{\alpha}} e_{i}, \ldots, A_{\xi_{\alpha}} e_{j}, \ldots, A_{\xi} e_{2 n}\right)\right) \\
= & (-2 n)(k-1) \operatorname{det} A_{\xi}+\sum_{i \neq j} \sum_{\alpha=1}^{k-1} \omega\left(A_{\xi} e_{1}, \ldots, A_{\xi_{\alpha}} e_{i}, \ldots, A_{\xi_{\alpha}} e_{j}, \ldots, A_{\xi} e_{2 n}\right) \\
= & ((-2 n)(k-1)-2 n(2 n-1)) \operatorname{det} A_{\xi} \\
& +\sum_{i \neq j} \sum_{\alpha=1}^{k} \omega\left(A_{\xi} e_{1}, \ldots, A_{\xi_{\alpha}} e_{i}, \ldots, A_{\xi_{\alpha}} e_{j}, \ldots, A_{\xi} e_{2 n}\right) \\
= & (-2 n)(k+2 n-2) \operatorname{det} A_{\xi}+\sum_{i \neq j} \sum_{\alpha=1}^{k} \omega\left(A_{\xi} e_{1}, \ldots, A_{\xi_{\alpha}} e_{i}, \ldots, A_{\xi_{\alpha}} e_{j}, \ldots, A_{\xi} e_{2 n}\right) .
\end{aligned}
$$




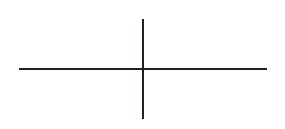

TOTAL CURVATURE OF COMPLETE SUBMANIFOLDS OF EUCLIDEAN SPACE

If we denote

$$
f_{i j}(\xi)=\sum_{\alpha=1}^{k} \omega\left(A_{\xi} e_{1}, \ldots, A_{\xi_{\alpha}} e_{i}, \ldots, A_{\xi_{\alpha}} e_{j}, \ldots, A_{\xi} e_{2 n}\right)
$$

then similarly we can compute

$$
\begin{aligned}
\Delta f_{i j}(\xi)= & (-2(n-1))(k+2(n-1)-2) f_{i j} \\
& +\sum_{\alpha, \beta} \sum_{r \neq s} \omega\left(A_{\xi} e_{1}, \ldots, A_{\xi_{\beta}} e_{r}, \ldots, A_{\xi_{\alpha}} e_{i}, \ldots, A_{\xi_{\alpha}} e_{j}, \ldots, A_{\xi_{\beta}} e_{s}, \ldots, A_{\xi} e_{2 n}\right) .
\end{aligned}
$$

If we continue this procedure and integrate over $\perp_{p}^{1}$, we obtain

$$
\int_{\perp_{p}^{1}} \operatorname{det} A_{\xi} d \xi=\frac{1}{(2 n)(k+2 n-2)} \frac{1}{2(n-1)(k+2(n-1)-2)} \cdots \frac{1}{2 k} H(p) c_{k-1},
$$

where

$$
H(p)=\sum_{\tau \in \sigma_{2 n}} \sum_{\alpha_{1}, \ldots, \alpha_{n}=1}^{k}(\operatorname{sgn} \tau) \omega\left(A_{\xi_{\alpha_{1}}} e_{\tau(1)}, A_{\xi_{\alpha_{1}}} e_{\tau(2)}, \ldots, A_{\xi_{\alpha_{n}}} e_{\tau(2 n-1)}, A_{\xi_{\alpha_{n}}} e_{\tau(2 n)}\right),
$$

which does not depend on the choice of an orthonormal basis, the first sum ranging over all permutations of $2 n$ elements. From the equation

$$
c_{m}=c_{2 n+k-1}=c_{2 n+k-3} \frac{c_{1}}{2 n+k-2}=\frac{\left(c_{1}\right)^{n}}{(2 n+k-2)(2 n+k-4) \cdots k} c_{k-1},
$$

we obtain that

$$
\int_{\perp_{p}^{1}} \operatorname{det} A_{\xi} d \xi=\frac{1}{2^{n} n !} \frac{c_{m}}{2^{n} \pi^{n}} H(p)
$$

Since

we can write this as

$$
2^{n} n !(2 \pi)^{n}=\frac{c_{2 n}}{2}(2 n) !
$$

$$
\frac{1}{c_{m}} \int_{\perp_{p}^{1}} \operatorname{det} A_{\xi} d \xi=\frac{2}{c_{2 n}(2 n) !} H(p) .
$$

We still have to prove that the right hand side of (4.1) is of intrinsic nature. Introducing the usual notation $h_{i j}^{\alpha}=\left\langle A_{\xi_{\alpha}} e_{i}, e_{j}\right\rangle$, we compute that

$$
H(p)=\sum_{\tau, \eta \in \sigma_{2 n}} \sum_{\alpha_{1}, \ldots, \alpha_{n}=1}^{k}(\operatorname{sgn} \tau)(\operatorname{sgn} \eta) h_{\tau(1) \eta(1)}^{\alpha_{1}} h_{\tau(2) \eta(2)}^{\alpha_{1}} \cdots h_{\tau(2 n-1) \eta(2 n-1)}^{\alpha_{n}} h_{\tau(2 n) \eta(2 n)}^{\alpha_{n}} .
$$

This can be rewritten as

$$
\begin{array}{r}
H(p)=\frac{1}{2^{n}} \sum_{\tau, \eta \in \sigma_{2 n}} \sum_{\alpha_{1}, \ldots, \alpha_{n}=1}^{k}(\operatorname{sgn} \tau)(\operatorname{sgn} \eta)\left(h_{\tau(1) \eta(1)}^{\alpha_{1}} h_{\tau(2) \eta(2)}^{\alpha_{1}}-h_{\tau(2) \eta(1)}^{\alpha_{1}} h_{\tau(1) \eta(2)}^{\alpha_{1}}\right) \cdots \\
\left(h_{\tau(2 n-1) \eta(2 n-1)}^{\alpha_{n}} h_{\tau(2 n) \eta(2 n)}^{\alpha_{n}}-h_{\tau(2 n) \eta(2 n-1)}^{\alpha_{n}} h_{\tau(2 n-1) \eta(2 n)}^{\alpha_{n}}\right),
\end{array}
$$




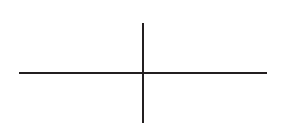

which, using the Gauss equation, amounts to

$$
H(p)=\frac{1}{(-2)^{n}} \sum_{\tau, \eta \in \sigma_{2 n}}(\operatorname{sgn} \tau)(\operatorname{sgn} \eta) R_{\tau(1) \tau(2) \eta(1) \eta(2)} \cdots R_{\tau(2 n-1) \tau(2 n) \eta(2 n-1) \eta(2 n)} .
$$

It is now clear that $H$ is an intrinsic invariant. Up to scaling, it is the usual Gauss-Bonnet integrand. If we put

$$
G(p)=\sum_{\tau, \eta \in \sigma_{2 n}}(\operatorname{sgn} \tau)(\operatorname{sgn} \eta) R_{\tau(1) \tau(2) \eta(1) \eta(2)} \cdots R_{\tau(2 n-1) \tau(2 n) \eta(2 n-1) \eta(2 n)},
$$

then

$$
H(p)=\frac{1}{(-2)^{n}} G(p) .
$$

Hence we have proved the following theorem.

THEOREM 4.1. Let $M^{2 n}$ be a submanifold in $\boldsymbol{E}^{m+1}$ and let $p \in M$. Then

$$
\frac{1}{c_{m}} \int_{\perp_{p}^{1}} \operatorname{det} A_{\xi} d \xi=\frac{2}{c_{2 n}(2 n) !(-2)^{n}} G(p)
$$

where $G$ is given by (4.2).

In particular, for surfaces this gives the following

COROLlary 4.2. Let $M^{2}$ be a surface in $\boldsymbol{E}^{m+1}$ and let $p \in M$. Then

$$
\frac{1}{c_{m}} \int_{\perp_{p}^{1}} \operatorname{det} A_{\xi} d \xi=\frac{1}{2 \pi} K(p),
$$

where $K$ is the Gauss curvature of $M^{2}$.

Looking at the proof of Theorem 4.1, we may notice that almost the same proof holds for submanifolds of Riemannian manifolds of constant sectional curvature, obtaining in this way the following theorem.

THEOREM 4.3. Let $M^{2 n}$ be a submanifold in a real space form $N^{m+1}(c)$ and let $p \in$ $M$. Then

where $G_{c}$ is given by

$$
\frac{1}{c_{m}} \int_{\perp_{p}^{1}} \operatorname{det} A_{\xi} d \xi=\frac{2}{c_{2 n}(2 n) !(-2)^{n}} G_{c}(p)
$$

$$
G_{c}(p)=\sum_{\tau, \eta \in \sigma_{2 n}}(\operatorname{sgn} \tau)(\operatorname{sgn} \eta)\left(R_{\tau(1) \tau(2) \eta(1) \eta(2)}-c\right) \cdots\left(R_{\tau(2 n-1) \tau(2 n) \eta(2 n-1) \eta(2 n)}-c\right) .
$$

For surfaces this again becomes the following:

THEOREM 4.4. Let $M^{2}$ be a surface in a real space form $N^{m+1}(c)$ and let $p \in M$. Then

$$
\frac{1}{c_{m}} \int_{\perp_{p}^{1}} \operatorname{det} A_{\xi} d \xi=\frac{1}{2 \pi}(K(p)-c) .
$$




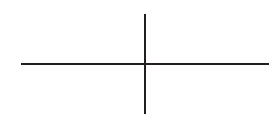

TOTAL CURVATURE OF COMPLETE SUBMANIFOLDS OF EUCLIDEAN SPACE

If now $M^{2 n}$ is compact and oriented, then integrating (4.1) and using Theorem (3.5), we obtain that

$$
\int_{M} G(p) d M=c_{2 n}(2 n) !(-1)^{n} 2^{n-1} \chi(M) .
$$

From the Nash embedding theorem one obtains the following corollary.

THEOREM 4.5 (Gauss-Bonnet-Chern). Let $M^{2 n}$ be a compact oriented Riemannian manifold, then

$$
\int_{M} G(p) d M=c_{2 n}(2 n) !(-1)^{n} 2^{n-1} \chi(M) .
$$

For an intrinsic proof compare [15] or [69, Sect.2.7].

4.2. The even elementary symmetric functions. Let $M^{n}$ be a submanifold in $\boldsymbol{E}^{m+1}=$ $\boldsymbol{E}^{n+k}$ and let $p \in M$. Let $\perp_{p}^{1}$ be the unit sphere in the normal space at $p$. Let $\omega$ be the volume element of $M$ at $p$, and let $\left\{e_{1}, \ldots, e_{n}\right\}$ be an orthonormal basis of $T_{p} M$. Let $l$ be any integer such that $1 \leq 2 l \leq n$. If $\xi \in \perp{ }_{p}^{1}$, then

$$
K_{2 l}(\xi)=\sum_{i_{1}<\cdots<i_{2 l}} \omega\left(e_{1}, \ldots, A_{\xi} e_{i_{1}}, \ldots, A_{\xi} e_{i_{2 l}}, \ldots, e_{n}\right) .
$$

Using the same method as above, we obtain

$$
\int_{\perp_{p}^{1}} K_{2 l}(\xi) d \xi=\frac{2 c_{2 l+k-1}}{c_{2 l}(2 l) !} H_{2 l}(p),
$$

where

$$
\begin{gathered}
H_{2 l}(p)=\sum_{i_{1}<\cdots<i_{2 l}} \sum_{\tau \in \sigma_{2 l} l} \sum_{\alpha_{1}, \ldots, \alpha_{l}=1}^{k}(\operatorname{sgn} \tau) \omega\left(e_{1}, \ldots, A_{\xi_{\alpha_{1}}} e_{i_{\tau(1)}}, \ldots, A_{\xi_{\alpha_{1}}} e_{i_{\tau(2)}}, \ldots,\right. \\
\left.A_{\xi_{\alpha_{l}}} e_{i_{\tau(2 l-1)}}, \ldots, A_{\xi_{\alpha_{l}}} e_{i_{\tau(2 l)}}, \ldots, e_{n}\right) .
\end{gathered}
$$

With the same notation as above we obtain

$$
H_{2 l}(p)=\sum_{i_{1}<\cdots<i_{2 l}} \sum_{\tau, \eta \in \sigma_{2 l} l} \sum_{\alpha_{1}, \ldots, \alpha_{l}=1}^{k}(\operatorname{sgn} \tau)(\operatorname{sgn} \eta) h_{i_{\tau}(1) i_{\eta(1)}}^{\alpha_{1}} h_{i_{\tau(2)} i_{\eta(2)}}^{\alpha_{1}} \cdots h_{i_{\tau}(2 l-1)}^{\alpha_{l}} i_{\eta(2 l-1)} h_{i_{\tau(2 l)} i_{\eta(2 l)}}^{\alpha_{l}},
$$

which, using the Gauss equation, can be written as

$$
H_{2 l}(p)=\frac{1}{(-2)^{l}} \sum_{i_{1}<\cdots<i_{2 l}} \sum_{\tau, \eta \in \sigma_{2 l}}(\operatorname{sgn} \tau)(\operatorname{sgn} \eta) R_{i_{\tau(1)} i_{\tau(2)} i_{\eta(1)} i_{\eta(2)}} \cdots R_{i_{\tau(2 l-1)} i_{\tau(2 l)} i_{\eta(2 l-1)} i_{\eta(2 l)}} .
$$

It is now clear that $H_{2 l}$ is an intrinsic invariant. If we introduce

$$
G_{2 l}(p)=\sum_{i_{1}<\cdots<i_{2 l}} \sum_{\tau, \eta \in \sigma_{2 l}}(\operatorname{sgn} \tau)(\operatorname{sgn} \eta) R_{i_{\tau(1)} i_{\tau(2)} i_{\eta(1)} i_{\eta(2)}} \cdots R_{i_{\tau(2 l-1)} i_{\tau(2 l)} i_{\eta(2 l-1)} i_{\eta(2 l)}},
$$

then

$$
H_{2 l}(p)=\frac{1}{(-2)^{l}} G_{2 l}(p) .
$$

Hence we have proved the following theorem. 


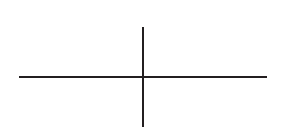

THEOREM 4.6. Let $M^{n}$ be a submanifold in $\boldsymbol{E}^{m+1}$, and let $p \in M$ and $l$ an integer such that $1<2 l \leq n$. Then,

$$
\frac{1}{c_{2 l+m-n}} \int_{\perp_{p}^{1}} K_{2 l}(\xi) d \xi=\frac{2}{c_{2 l}(2 l) !(-2)^{l}} G_{2 l}(p),
$$

where $G_{2 l}$ is given by (4.5)

Similarly, this carries over to the case of submanifolds in real space forms as follows.

THEOREM 4.7. Let $M^{n}$ be a submanifold in a real space form $N^{m+1}(c)$, and let $p \in M$ and $l$ an integer such that $1<2 l \leq n$. Then

$$
\frac{1}{c_{2 l+m-n}} \int_{\perp_{p}^{1}} K_{2 l}(\xi) d \xi=\frac{2}{c_{2 l}(2 l) !(-2)^{l}} G_{2 l, c}(p),
$$

where $G_{2 l, c}$ is given by

$$
G_{2 l, c}(p)=\sum_{i_{1}<\cdots<i_{2 l}} \sum_{\tau, \eta \in \sigma_{2 l}}(\operatorname{sgn} \tau)(\operatorname{sgn} \eta)\left(R_{i_{\tau(1)} i_{\tau(2)} i_{\eta(1)} i_{\eta(2)}}-c\right) \cdots\left(R_{i_{\tau(2 l-1)} i_{\tau(2 l)} i_{\eta(2 l-1)} i_{\eta(2 l)}}-c\right) .
$$

5. Limit directions of complete open submanifolds and submanifolds with conelike ends. It was the idea of Wintgen [70] to study the total curvature and total absolute curvature of complete open submanifolds in $\boldsymbol{E}^{m+1}$ by means of limit directions. A unit vector $e \in S^{m}$ is called a limit direction if there is a sequence $\left(p_{n}\right)_{n \in N}$ of points in $M$ converging to one particular end such that

$$
e=\lim _{n \rightarrow \infty} \frac{p_{n}}{\left\|p_{n}\right\|} .
$$

The set of all limit directions of $M$ is denoted by $M_{\infty}$. One of Wintgen's results states that the Gauss-Bonnet theorem

$$
\int_{\perp^{1}} K d V_{\text {can }}=c_{m} \chi(M)
$$

holds if $M$ is even-dimensional, if $K$ is absolutely integrable and if there are only finitely many limit directions. Especially, this set $M_{\infty}$ of limit directions in $S^{m}$ provides an extrinsic analogue of the ideal boundary, provided that $M_{\infty}$ has a reasonable structure, e.g., as a smooth submanifold of lower dimension.

DEFINITION 5.1 (Conical end). Let $M_{\infty} \subset S^{m}(1)$ be a compact $(n-1)$-dimensional submanifold. Then for fixed $p \in \boldsymbol{E}^{m+1}$ the set $C\left(M_{\infty}\right):=\left\{p+t \cdot x \mid x \in M_{\infty}, t \geq 0\right\} \subset \boldsymbol{E}^{m+1}$ is called the (simple) cone over $M_{\infty}$ with apex $p$. An $n$-dimensional complete submanifold $M$ of $\boldsymbol{E}^{m+1}$ with finitely many ends is said to have conical ends if for a certain radius $R>0$ the set $M \backslash B^{m+1}(R)$ consists only of the union of open subsets of cones, where the apex may vary from one end to another. In this case, $M \backslash B^{m+1}(R)$ is the union of open subsets of cones over the components of $M_{\infty}$, each counted with multiplicity. 


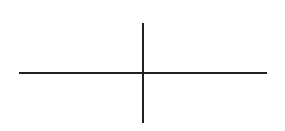

Proposition 5.2. For a complete submanifold $M^{n} \subset \boldsymbol{E}^{m+1}$ with conical ends the Gauss-Bonnet defect equals the total outer curvature of $M_{\infty} \subset S^{m}$, where one has to sum up over the set of ends separately:

$$
c_{m} \chi(M)-\int_{\perp^{1}(M)} K d V_{\mathrm{can}}=\sum_{0 \leq 2 i \leq n-1} \frac{c_{m}}{c_{m-n+2 i} c_{n-1-2 i}} K_{2 i}\left(M_{\infty}\right)
$$

where $\boldsymbol{K}_{j}\left(M_{\infty}\right)=\int_{\perp^{1}\left(M_{\infty}\right)} K_{j} d V_{\text {can }}$ denotes the total $j^{\text {th }}$ curvature of the set $M_{\infty}$ (for each end separately), regarded as a submanifold of the unit sphere.

First of all, the total curvature converges, since the curvature is zero on the cones, i.e., outside a compact set. If the apex of each cone is the origin, the assertion follows from Theorem 3.9 because the total curvature is scale invariant (thus we can assume $R=1$ ) and because the various cones have vanishing curvature, i.e., the total curvature of $M$ equals the total curvature of $M \cap B^{m+1}$ (1). If an apex is not the origin, then we can use the fact that by the Gauss-Bonnet formula the total curvature is invariant under changes in a compact part and that it is also invariant under translations of the cones. This implies that the total curvature and the right hand side of the equation in 5.2 behaves like in the case where each apex is the origin.

For this argument it is not necessary that the ends are exactly cones. We have the same geometric phenomenon if the ends are (asymptotically) cone-like in a sense to be made more precise below. Recall that the following characteristic property of a cone $C$ over a manifold with apex 0: All the intersections with $S^{m}(R)$ are homothetic to one another, tangent and normal spaces at corresponding points are parallel to each other, and at each point the outer normal of $C \cap B^{m+1}$ coincides with the position vector (up to scaling).

Definition 5.3 (Cone-like end). An end $E$ of a complete submanifold $M^{n} \subset \boldsymbol{E}^{m+1}$ with associated component $M_{\infty}^{E}$ in the set of limit directions is said to be (asymptotically) cone-like if the following conditions are satisfied:

(1) There is a point $q$ such that for sufficiently large $R$ the intersection $E \cap S^{m}(R ; q)$ is an $(n-1)$-dimensional submanifold of the sphere of radius $R$ around $q$, and

$$
\lim _{R \rightarrow \infty} \frac{1}{R}\left(E \cap S^{m}(R ; q)\right)=M_{\infty}^{E}
$$

in the $C^{2}$-topology. This property is actually independent of the choice of $q$, so that we may assume that $q$ is the origin 0 .

(2) For every $\varepsilon$ there is a number $R_{0}$ such that for each $R>R_{0}$ the angle between outer unit normal of the submanifold $E \cap B^{m+1}(R ; 0)$ at any point $p \in E,\|p\|=R$, and the position vector $p$ is at most $\varepsilon$.

THEOREM 5.4. For a complete submanifold $M^{n} \subset \boldsymbol{E}^{m+1}$ with finitely many cone-like ends the Gauss-Bonnet defect is given by the same formula for $M_{\infty} \subset S^{m}$ as in Proposition 


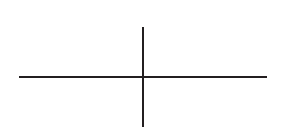

5.2:

$$
c_{m} \chi(M)-\int_{\perp^{1}} K d V_{\mathrm{can}}=\sum_{0 \leq 2 i \leq n-1} \frac{c_{m}}{c_{m-n+2 i} c_{n-1-2 i}} \boldsymbol{K}_{2 i}\left(M_{\infty}\right) .
$$

If we divide the equation (5.1) by $c_{m}$, we see from Theorem 4.1 that the left hand side of (5.1) is intrinsic for $M$, and from Theorem 4.7 we obtain that the right hand side is intrinsic for $M_{\infty}$. The right hand side was called the total outer curvature in [27].

PROOF. The proof follows from the extrinsic Gauss-Bonnet formula 3.9 and Proposition 5.2 above. For sufficiently large $R$ the subspaces

$$
M_{R}:=M \cap B^{m+1}(R ; 0)
$$

are diffeomorphic to one another. We would like to apply Theorem 3.9 to $M_{R}$ but this is not literally possible since it is not orthogonal at the boundary in $S^{m}(R ; 0)$. However, by Definition 5.3 the tangent and normal space of $M_{R}$ converges to the tangent and normal space of $M_{\infty}$, and in the limit the orthogonality is satisfied. This implies that Theorem 3.9 holds for $M_{R}$ in the limit $R \rightarrow \infty$. Because of scale invariance the total curvature of the interior of $M_{R}$ converges to the total curvature of $M$ for $R \rightarrow \infty$. By Definition 5.3 the total outer curvature of $M_{R}$ converges to the total outer curvature of $M_{\infty}$. Thus the formula in Proposition 5.2 can be applied. As an appropriate geometric picture, we can think of $M$ as sitting in the unit ball with the same boundary behavior as in Theorem 3.9, together with a cone over the boundary to infinity. This cone has vanishing Lipschitz-Killing curvature and thus does not contribute to the total curvature. Note, however, that for $R \rightarrow \infty$ the "sequence" $(1 / R) M_{R}$ does not converge to a smooth submanifold but rather to a cone over $M_{\infty}$. For 2-dimensional surfaces this is also intrinsically true, see [42].

COROLlary 5.5. (1) If in addition all curvatures $K_{2 i}$ of $M_{\infty}$ are nonnegative, then the Cohn-Vossen inequality holds.

(2) If in addition for each end $M_{\infty}^{E}$ is totally geodesic in $S^{m}$, then we have

$$
\chi(M)-\frac{1}{c_{m}} \int_{\perp^{1}} K d V=k,
$$

where $k$ denotes the number of ends.

COROLlARY 5.6. For a 2-dimensional open surface $M^{2} \subset \boldsymbol{E}^{m+1}$ with cone-like ends we have

$$
c_{m} \chi(M)-\int_{\perp^{1}} K_{2} d V_{\text {can }}=\frac{c_{m}}{2 \pi} \operatorname{length}\left(M_{\infty}\right) \geq 0 .
$$

From Corollary 4.2 we the obtain the following.

COROLlARY 5.7. For a 2-dimensional open surface $M^{2} \subset \boldsymbol{E}^{3}$ with cone-like ends the Gauss-Bonnet defect equals the total length of $M_{\infty} \subset S^{2}$ (counted with multiplicity, i.e., for each end separately) :

$$
2 \pi \chi(M)-\int_{M} K d A=\operatorname{length}\left(M_{\infty}\right) \geq 0,
$$




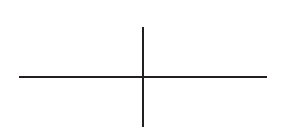

TOTAL CURVATURE OF COMPLETE SUBMANIFOLDS OF EUCLIDEAN SPACE

where $K$ is the Gauss curvature. This implies the Cohn-Vossen inequality.

COROLLARY 5.8 ([27]). For an open hypersurface $M^{4} \subset \boldsymbol{E}^{5}$ with cone-like ends the Gauss-Bonnet defect is

$$
\frac{4}{3} \pi^{2} \chi(M)-\int_{M} K_{4} d V_{M}=\frac{1}{6} \int_{M_{\infty}}(S-2) d V_{M_{\infty}},
$$

where the integral has to be taken for each end separately.

The proof follows directly from Theorem 5.4 and Corollary 3.10.

COROLlARY 5.9. For a 4-dimensional complete open hypersurface with cone-like ends the Gauss-Bonnet equality holds if and only if the average (= integral mean) of the scalar curvature of the ideal boundary in $S^{4}$ is 2 . The Cohn-Vossen inequality remains valid if and only if this average is greater than or equal to 2.

Under the assumption of nonnegative sectional curvature the validity of the Cohn-Vossen inequality was established in [65] for hypersurfaces and in [39] for codimension two. By a theorem of Sacksteder [51] and Wu [71], such a hypersurface is necessarily convex. Then the total curvature ranges between 0 and $c_{m} / 2$, just as in the classical case for surfaces with positive Gauss curvature in Theorem 2.1.

Note that the value 2 for the scalar curvature has a special meaning by the following gap theorem: It is known that a compact hypersurface of $S^{4}(1)$ with constant mean curvature and constant scalar curvature can satisfy $S \leq 2$ only if it is a member of Cartan's isoparametric family of hypersurface with $S=0$, see [4], [12]. The other examples with constant mean curvature and constant scalar curvature are the round 3 -spheres and the products $S^{1}(r) \times$ $S^{2}\left(\sqrt{1-r^{2}}\right)$, the latter ones satisfy $S>2$ and $\lim _{r \rightarrow 0} S=2$, compare Corollary 5.12 below.

COROLlARY 5.10. The Cohn-Vossen inequality does not hold in general for complete open 4-dimensional hypersurfaces in Euclidean 5-space.

This can be seen from the following key example with vanishing scalar curvature at infinity. It is mentioned in [10] that certain examples have been constructed, and in [47] an example is intrinsically given, where the end involves a flat $(2 n-1)$-torus. The following example seems to be quite simple.

EXAMPLE 5.11 (Key Example: Cone over Cartan's hypersurface). Let $x: \boldsymbol{R} P^{2} \rightarrow S^{4}$ be the Veronese surface. The family of tubes around it defines an isoparametric family [11, pp. 296-299], in particular, the tube with radius $\pi / 2$ is Cartan's minimal isoparametric hypersurface with principal curvatures $\sqrt{3}, 0,-\sqrt{3}$, and hence

$$
K_{1}=0, K_{2}=-3, S=6+2 K_{2}=0 .
$$

However, one has $S=0$ for each member in the whole isoparametric family. Furthermore, note that in this special case the tube with radius $\pi / 6$ coincides with Cartan's isoparametric hypersurface, so that the entire 4-sphere decomposes into the two $(\pi / 6)$-tubes as disc bundles over the Veronese surface and its antipodal copy. Let now $X \subset S^{4}$ be a solid open tube around 


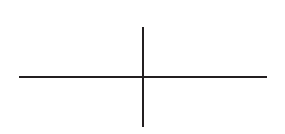

the Veronese surface with radius $\pi / 6$, bounded by Cartan's isoparametric hypersurface. We define an embedding $F: X \rightarrow \boldsymbol{R}^{5}$ locally by

$$
F(s, t, p):=\varphi(t)(\cos (\psi(t)) \cdot x(p)+\sin (\psi(t)) \cdot(\cos s \cdot \xi(p)+\sin s \cdot \eta(p))),
$$

where $s, t \geq 0, p \in \boldsymbol{R} P^{2}$. Here $\xi, \eta$ denote orthogonal unit normal vector fields to $x$, and $\varphi, \psi$ are smooth real functions as follows: An even function

$$
\varphi:[0, \infty) \rightarrow[0, \infty)
$$

such that $\varphi(0)>0, \varphi^{\prime}(0)=0, \varphi^{\prime}>0$ otherwise, $\varphi(t)=t+1-\pi / 6$ for $t \geq \pi / 6$, and an odd function

$$
\psi:[0, \infty) \rightarrow[0, \pi / 6]
$$

such that $\psi(0)=0, \psi^{\prime}(0)=1, \psi^{\prime}(t)>0$ for $t<\pi / 6, \psi(t)=\pi / 6$ for $t \geq \pi / 6$. Then $F(s, 0, p)=\varphi(0) \cdot x(p)$ is a scaled copy of the Veronese surface and $F(s, \pi / 6, p)$ describes Cartan's hypersurface. This is true even though $\xi, \eta$ are defined only locally. In different local charts the various definitions fit together. For $t \geq \pi / 6$ the mapping $F$ describes a cone over Cartan's hypersurface, and hence $F$ (or rather its image $F(X)$ ) has one conical end. The corresponding $X_{\infty}$ is Cartan's hypersurface itself with vanishing scalar curvature and nonvanishing volume. This implies that the Gauss-Bonnet defect is strictly negative, according to Corollary 5.9.

COROLlary 5.12. The (strict) Cohn-Vossen inequality holds for 4-dimensional hypersurfaces with cone-like ends if each end is of the type "cone over a round sphere $S^{3}(r)$ " or "cone over a Clifford torus $S^{1}(r) \times S^{2}\left(\sqrt{1-r^{2}}\right)$ ".

PROOF. If the end is of type $S^{3}(r), 0<r \leq 1$, then the scalar curvature is $S=6 r^{-2}$; the volume element is $r^{3} d V_{S^{3}(1)}$, and so the Gauss-Bonnet defect of this end turns out to be

$$
\frac{1}{3} \int_{S^{3}}\left(6 r^{-2}-2\right) r^{3} d V_{S^{3}}=\frac{2}{3} \pi^{2} r\left(6-2 r^{2}\right)>0 .
$$

For $r=1$ we obtain the value $8 \pi^{2} / 3=c_{4}$, compare Corollary 5.5 above. In the limit $r \rightarrow 0$ we obtain a vanishing Gauss-Bonnet defect, in accordance with [70].

If the end is of the type of the Clifford torus with $0<r<1$, then we have $S=2 /\left(1-r^{2}\right)$, the volume element is $r\left(1-r^{2}\right) d V_{S^{1}(1)} \wedge d V_{S^{2}(1)}$, and so the Gauss-Bonnet defect is

$$
\frac{1}{3} \int_{S^{1} \times S^{2}}\left(\frac{2}{1-r^{2}}-2\right) r\left(1-r^{2}\right) d V_{S^{1}} \wedge d V_{S^{2}}=\frac{16}{3} \pi^{2} r^{3}>0 .
$$

The case of spherically-symmetric ends was also discussed in [47, p. 329].

6. The variational problem for the total curvature. The variational problem for various curvature functionals has been studied during many years. One of the important results is certainly the theorem of Hilbert stating that intrinsically the gradient of the total scalar curvature functional (the Hilbert-Einstein functional) within the class of Riemannian metrics on a given manifold $M$ is nothing but the Einstein tensor $(S / 2) g-$ Ric, see $[9$, Sect. 4C]. The gradient of the area functional within a family of metrics $g_{t}=g+t \cdot h$ is known to be half 


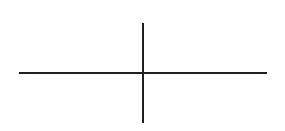

TOTAL CURVATURE OF COMPLETE SUBMANIFOLDS OF EUCLIDEAN SPACE

of the trace of $h$. In the classical case of surfaces in Euclidean 3-space or the 3-sphere this gradient is nothing but the mean curvature of the surface. This had tremendous influence to differential geometry and analysis, and the stationary surfaces (called minimal surfaces) have permanently been a field of intensive and extensive research. The variation of the extrinsic higher mean curvature functionals

$$
\boldsymbol{K}_{i}(M)=\int_{M} K_{i} d V_{M}
$$

was studied much later. As in Theorem 3.9, here $K_{i}$ denotes the $i^{\text {th }}$ elementary symmetric function of the eigenvalues of the shape operator $A$ of a hypersurface. The normalization is chosen such that the characteristic polynomial is $\operatorname{det}(A+\lambda \cdot \mathrm{Id})=\sum_{i} K_{i} \lambda^{n-i}$ if $M$ is $n$-dimensional. In terms of the principal curvatures $\kappa_{i}$ one has $K_{i}=\sum_{j_{1}<\cdots<j_{i}} \kappa_{j_{1}} \kappa_{j_{2}} \cdots \kappa_{j_{i}}$.

THEOREM 6.1 (K. Voss [63], compare [46, 48]). For any hypersurface in Euclidean space the gradient of the $i^{\text {th }}$ curvature functional $\boldsymbol{K}_{i}=\int K_{i} d V$ is the function $-(i+1) K_{i+1}$.

COROLLARY 6.2. The Hilbert-Einstein functional for a hypersurface in $\boldsymbol{E}^{n+1}$ is stationary within the class of hypersurfaces if and only if $K_{3} \equiv 0$.

REMARK 6.3. By a result of Fialkow [25] any Einstein hypersurfaces in Euclidean space is either totally umbilical or developable. In particular, it has constant sectional curvature in any case.

REMARK 6.4. For $n=3$ this condition $K_{3}=0$ just means that the rank of the shape operator is at most 2 . For any $n \geq 4$ there are non-developable examples of complete hypersurfaces satisfying $K_{3}=0$ as follows: If a planar curve with curvature $\kappa$ rotates in $(n+1)$ space, then the principal curvatures are $\kappa$ and a certain $\lambda$ of multiplicity $n-1$. Then

$$
K_{3}=\left(\begin{array}{c}
n-1 \\
3
\end{array}\right) \lambda^{3}+\left(\begin{array}{c}
n-1 \\
2
\end{array}\right) \kappa \lambda^{2}=\left(\begin{array}{c}
n-1 \\
2
\end{array}\right) \lambda^{2}\left(\frac{n-3}{3} \lambda+\kappa\right) .
$$

Hence we have $K_{3}=0$ if the quotient $\lambda / \kappa$ equals the constant $-3 /(n-3)$. Such curves have been investigated and explicitly determined by Hopf in [33] for the study of rotational surfaces with a linear relation between the two principal curvatures. The resulting surfaces and hypersurfaces are complete and analytic.

THEOREM 6.5 (Reilly [48], compare [64]). For a hypersurface in the unit n-sphere the gradient of the curvature functional $\boldsymbol{K}_{i}=\int K_{i} d V$ is the function $-(i+1) K_{i+1}+(n-i) K_{i-1}$.

If we compare this to the Hilbert-Einstein functional, we obtain the following: Since the scalar curvature $S$ is the sum of all sectional curvatures $K_{i j}(i \neq j)$, the Gauss equation

$$
K_{i j}=1+\kappa_{i} \kappa_{j}
$$

leads to

$$
S=n(n-1)+\sum_{i \neq j} \kappa_{i} \kappa_{j}=n(n-1) K_{0}+2 K_{2} .
$$




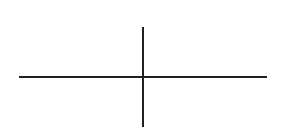

Hence the gradient of the total scalar curvature functional is

$$
n(n-1)\left(-K_{1}\right)+2\left(-3 K_{3}+(n-2) K_{1}\right)=-6 K_{3}+\left(-n^{2}+3 n-4\right) K_{1} .
$$

COROLLARY 6.6. The Hilbert-Einstein functional for a hypersurface in $S^{n}(1)$ is stationary within the class of hypersurfaces if and only if $3 K_{3}+\left(\left(\begin{array}{c}n-1 \\ 2\end{array}\right)+1\right) K_{1} \equiv 0$.

One example satisfying this equation is Cartan's isoparametric hypersurface in $S^{4}$, see the key example 5.11 .

THEOREM 6.7. For even $n$ the gradient of the total outer curvature functional (= the right hand side in Theorem 3.9) of a hypersurface in $S^{n}$ is the negative Gauss-Kronecker curvature $-K_{n-1}$ of this hypersurface.

PROOF. If $\delta$ denotes the gradient, then we have $\delta \boldsymbol{K}_{i}=-(i+1) K_{i+1}+(n-i) K_{i-1}$ by Theorem 6.5 above. If $n$ is even, this implies

$$
\begin{aligned}
& \delta\left(\sum_{0 \leq 2 i \leq n-1} \frac{c_{n}}{c_{2 i} c_{n-1-2 i}} \boldsymbol{K}_{2 i}\right) \\
& =\sum_{0 \leq 2 i \leq n-2} \frac{c_{n}}{c_{2 i} c_{n-1-2 i}}\left(-(2 i+1) K_{2 i+1}+(n-2 i) K_{2 i-1}\right) \\
& =-\frac{c_{n}}{c_{n-2} c_{1}}(n-1) K_{n-1} \\
& \quad+\sum_{0 \leq 2 i \leq n-4} c_{n}\left(\frac{n-2 i-2}{c_{2 i+2} c_{n-3-2 i}}-\frac{2 i+1}{c_{2 i} c_{n-1-2 i}}\right) K_{2 i+1}=-K_{n-1} .
\end{aligned}
$$

In the last step we used the equation

$$
(j-1) c_{j}=c_{1} c_{j-2}
$$

which holds for arbitrary $j$.

REMARK 6.8. If $n$ is odd, then the same calculation shows that the gradient vanishes identically because the leading term $K_{n}$ vanishes on the $(n-1)$-dimensional boundary. This is not surprising, since we know from Corollary 3.8 that in this case the total curvature is constant, namely, the Euler characteristic.

COROLlaRY 6.9. The total curvature $\int_{M} K_{n} d V$ of an even-dimensional open hypersurface $M \subset \boldsymbol{E}^{n+1}$ with cone-like ends is stationary (within the class of such hypersurfaces having cone-like ends) if and only if each component of $M_{\infty}$ has vanishing Gauss-Kronecker curvature in the sphere "at infinity" or, equivalently, if it has one vanishing principal curvature at each point.

This follows from Theorem 6.7 and Theorem 5.4 because the gradient of $2 \int_{M} K_{n} d V$ is the function $K_{n-1}$ on $M_{\infty}$. Note that for $n=2$ the Gauss-Kronecker curvature of $M_{\infty}$ is nothing but the geodesic curvature of the boundary curve. Thus in the stationary 2-dimensional 


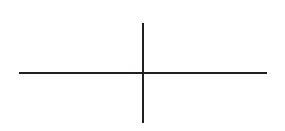

TOTAL CURVATURE OF COMPLETE SUBMANIFOLDS OF EUCLIDEAN SPACE

case we have the same behavior as in Osserman's formula for minimal surfaces: The GaussBonnet defect equals $2 \pi$ times the number of ends. Note that the total curvature is stationary for the key example 5.11. Corollary 6.9 raises the question what we can say about compact hypersurfaces of even-dimensional spheres with vanishing Gauss-Kronecker curvature.

7. Hypersurfaces of $S^{n+1}$ with vanishing Gauss-Kronecker curvature, and the quantization of the total curvature. It seems that not too much is known about compact hypersurfaces of the standard sphere with vanishing Gauss-Kronecker curvature. The case of constant intermediate curvature $K_{i}$ is treated in [66] but the case of $K_{n}=0$ is excluded there. In [23, Theorem 2] the following is shown: If the nullity index $v(x)$ of the second fundamental form at any point $x$ is always greater than a certain invariant $v_{n}$, then the submanifold is totally geodesic. Since in our case we assume $v(x) \geq 1$, we obtain this conclusion if $v_{n}=0$. For certain even values it is shown that indeed one has $v_{n}=0$. In particular, this holds if $n$ is a power of 2. However, in our case the variational problem in Section 6 is only interesting for odd $n$.

In this section we examine the situation in particular for $n=3$.

Definition 7.1 (Tube of radius $\pi / 2$ ). Let $\Sigma: N^{k} \rightarrow S^{n+1}(1), k<n$, be an isometric immersion and let $\perp^{1}(N)$ be the unit normal bundle. Then the tube of radius $\pi / 2$ over $\Sigma$ is defined as (the image of)

$$
x: \perp^{1}(N) \rightarrow S^{n+1}(1):(p, \xi) \mapsto \xi .
$$

LEMMA 7.2. The tube of radius $\pi / 2$ is an immersion if for each normal vector to $N$ the shape operator of $\Sigma$ is nondegenerate. If the tube is an immersion, then its Gauss-Kronecker curvature vanishes identically.

Proof. Locally $\perp^{1} N$ is the product of $N^{k}$ and $S^{n-k}(1)$. If $p \in N$ and $\xi$ is a unit normal to $N$ at $p$, then the tangent space to $\perp^{1}(N)$ at $(p, \xi)$ can be identified with $T_{p} N \times$ $T_{\xi} S^{n-k}(1)$. Let $v \in T_{p} N$ and $X \in T_{\xi} S^{n-k}(1)$, then

$$
x_{*}(v)=-\Sigma_{*}\left(A_{\xi}(v)\right)+\nabla_{v}^{\perp} \xi
$$

where $\nabla^{\perp}$ is the normal connection of $\Sigma$ and $A_{\xi}$ is the shape operator of $\Sigma$ with respect to $\xi$, and

$$
x_{*}(X)=X \text {. }
$$

Therefore $x$ is an immersion if and only if for each $\xi$ the shape operator $A_{\xi}$ is nondegenerate.

It also follows that

$$
N(p, \xi):=-\Sigma(p)
$$

is a unit normal vector to $x$ at $(p, \xi)$, the minus sign being taken to obtain the outer normal. Let $A$ denote the shape operator of $x$ with respect to $N$. Then

$$
x_{*}(A v)=\Sigma_{*}(v)
$$

and

$$
x_{*}(A X)=0 .
$$




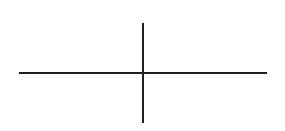

Therefore we conclude that $\operatorname{det} A=0$, and so every tube of radius $\pi / 2$ has vanishing GaussKronecker curvature.

Moreover, we have

$$
\begin{aligned}
x_{*}(A v) & =\Sigma_{*}(v)=\Sigma_{*}\left(A_{\xi} A_{\xi}^{-1} v\right) \\
& =-x_{*}\left(A_{\xi}^{-1} v\right)+\nabla_{A_{\xi}^{-1} v}^{\perp} \xi \\
& =-x_{*}\left(A_{\xi}^{-1} v\right)+x_{*}\left(\nabla_{A_{\xi}^{-1} v}^{\perp} \xi\right),
\end{aligned}
$$

and hence

$$
A(v)=-A_{\xi}^{-1} v+\nabla_{A_{\xi}^{-1} v}^{\perp} \xi
$$

This equation implies that the $k$-th elementary symmetric function of $A$ is given by

$$
K_{k}=(-1)^{k}\left(\operatorname{det} A_{\xi}\right)^{-1} \text {. }
$$

LEMMA 7.3. The volume element $d V$ of the tube $x$ of radius $\pi / 2$ satisfies

$$
d V=(\operatorname{det} A \xi) d V_{\text {can }}
$$

at each point $(p, \xi)$.

Proof. Let $\omega$ be the volume element of $\boldsymbol{R}^{n+2}$. Then, writing $v_{j}$ for vectors tangent to $N$ and $X_{j}$ for vectors tangent to $S^{n-k}(1)$,

$$
\begin{aligned}
(d V) & \left(v_{1}, \ldots, v_{k}, X_{1}, \ldots, X_{n-k}\right) \\
& =\omega\left(x(p), N(p, \xi), x_{*} v_{1}, \ldots, x_{*} v_{k}, x_{*} X_{1}, \ldots, x_{*} X_{n-k}\right) \\
& =\omega\left(\xi,-\Sigma(p),-\Sigma_{*} A_{\xi} v_{1}, \ldots,-\Sigma_{*} A_{\xi} v_{k}, X_{1}, \ldots, X_{n-k}\right) \\
& =\omega\left(\Sigma(p), \Sigma_{*} A_{\xi} v_{1}, \ldots, \Sigma_{*} A_{\xi} v_{k}, \xi, X_{1}, \ldots, X_{n-k}\right) \\
& =\left(\operatorname{det} A_{\xi}\right)\left(d V_{\text {can }}\right)\left(v_{1}, \ldots, v_{k}, X_{1}, \ldots, X_{n-k}\right)
\end{aligned}
$$

which proves the assertion.

Let us now study the special case of a 3-dimensional hypersurface of the 4-sphere.

THEOREM 7.4. Let $M^{3}$ be a compact hypersurface of $S^{4}(1)$ with vanishing GaussKronecker curvature. Assume that the rank of the shape operator is constant. Then

$$
\frac{1}{8 \pi^{2}} \int_{M}(S-2) d V \in \boldsymbol{Z} .
$$

PROOF. If $M^{3}$ is totally geodesic, then $S=6$, vol $M=2 \pi^{2}$ and the proof is finished. From [22] it follows that the rank of the shape operator cannot be 1, so that we can assume that the rank is 2 . Then $M^{3}$ is a tube over an immersed surface $N$ and we can apply the formulas obtained above. The Gauss equation for $M$ implies that the scalar curvature of $M$ is given by $S=6+2 K_{2}$, so that

$$
(S-2) d V=4 d V+2 K_{2} d V=4\left(1 / K_{2}\right) d V_{\text {can }}+2 d V_{\text {can }} .
$$




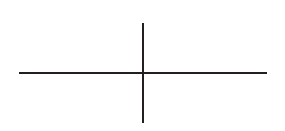

TOTAL CURVATURE OF COMPLETE SUBMANIFOLDS OF EUCLIDEAN SPACE

Under the assumptions we know that $K_{2}$ is nowhere zero. Let $\varepsilon$ be the sign of $K_{2}$. Then

$$
\begin{aligned}
\int_{M}(S-2) d V & =4 \int_{\perp^{1}(N)}\left(1 / K_{2}\right) d V_{\mathrm{can}}+2 \int_{\perp^{1}(N)} d V_{\mathrm{can}} \\
& =4 \int_{\perp^{1}(N)} \operatorname{det} A_{\xi} d V_{\mathrm{can}}+4 \pi \varepsilon \operatorname{vol} N \\
& =4 \varepsilon \int_{N} \pi(K-1) d N+4 \pi \varepsilon \operatorname{vol} N \\
& =8 \pi^{2} \varepsilon \chi(N),
\end{aligned}
$$

where we have used Theorem 4.4 and the ordinary Gauss-Bonnet theorem for $N$.

REMARK 7.5. Under the assumptions of the theorem above the topology of the 3dimensional hypersurface is essentially unique: Either it is totally geodesic and thus an equatorial 3-sphere or it must be diffeomorphic to Cartan's isoparametric hypersurface, according to [37]. However, the geometry is quite flexible in this case. One can slightly perturb the Veronese surface and then consider the tube around it of radius $\pi / 2$.

Finally we return to the investigation of the total curvature of complete hypersurfaces of Euclidean space. If we combine the previous theorem with Corollary 3.10, we obtain the following result.

THEOREM 7.6 (Quantization of the total curvature). Let $M^{4}$ be a complete open hypersurface of $\boldsymbol{E}^{5}$ with finitely many cone-like ends and with stationary total curvature. Assume that for each end the rank of the shape operator in the sphere "at infinity" is constant. Then the normalized total curvature takes values in the integers:

$$
\frac{3}{4 \pi^{2}} \int_{M} K_{4} d V \in Z
$$

This theorem can be considered as a kind of quantization of the total curvature for hypersurfaces with cone-like ends, under the additional condition that the total curvature is stationary (or, equivalently, that the Gauss-Kronecker curvature at infinity vanishes) and a condition on the rank of the shape operator, which we conjecture to be superfluous. This conjecture is formulated at the end of Section 1.

We remark that the conjecture holds for $n=2$. Indeed, in that case each end is a great circle, such that the length of $M_{\infty}$ is a multiple of $2 \pi$. Corollary 5.7 implies that $(1 / 2 \pi) \int_{M} K d V$ is an integer.

QUESTIONS. 1. One of the open questions is whether or not every compact hypersurface in the sphere with vanishing Gauss-Kronecker curvature is a $\pi / 2$-tube around some other submanifold. If yes, then this would provide a strategy for proving the conjecture on the quantization of the total curvature.

2. Since the Gauss-Bonnet difference term can be expressed by intrinsic curvatures $K_{2 i}$ of $M_{\infty}$ according to Theorem 5.4, the question arises whether this difference can be described 


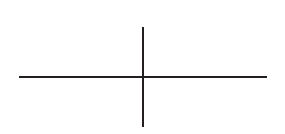

purely intrinsically in the original manifold $M$. For 4-dimensional complete Riemannian manifolds one would have to introduce a volume and an appropriate version of a scalar curvature of the ideal boundary "at infinity".

\section{REFERENCES}

[ 1 ] C. B. Allendoerfer, The Euler number of a Riemannian manifold, Amer. J. Math. 62 (1940), $243-248$.

[2] C. B. Allendoerfer And A. Weil, The Gauss-Bonnet theorem for Riemannian polyhedra, Trans. Amer. Math. Soc. 53 (1943), 101-129.

[3] S. C. DE Almeida And F. BRito, Minimal hypersurfaces of $S^{4}$ with constant Gauss-Kronecker curvature, Math. Z. 195 (1987), 99-107.

[4] S. C. DE Almeida And F. BRito, Closed 3-dimensional hypersurfaces with constant mean curvature and constant scalar curvature, Duke Math. J. 61 (1990), 195-206.

[ 5] S. C. DE Almeida And F. BRito, Closed hypersurfaces of $S^{4}$ with two constant symmetric curvatures, Ann. Fac. Sci. Toulouse Math. (6) 6 (1997), 187-202.

[6] W. Ballmann, M. Gromov and V. Schroeder, Manifolds of nonpositive curvature, Progr. Math. 61, Birkhäuser Boston, Inc., Boston, Mass., 1985.

[7] T. F. BANCHOFF AND W. KÜHNEL, Tight submanifolds, smooth and polyhedral, Tight and taut submanifolds (Berkeley, Calf., 1994), 51-118, Math. Sci. Res. Inst. Publ. 32, Cambridge Univ. Press, Cambridge, 1997.

[ 8 ] V. BAngERT, Total curvature and the topology of complete surfaces, Compos. Math. 41 (1980), 95-105.

[9] A. BESSE, Einstein Manifolds, Ergeb. Math. Grenzgeb. (3), Springer-Verlag, Berlin, 1987.

[10] D. BleEcker, The Gauss-Bonnet inequality and almost-geodesic loops, Adv. Math. 14 (1974), $183-193$.

[11] T. E. CECiL And P. J. Ryan, Tight and taut immersions of manifolds, Res. Notes Math. 107, Pitman, Boston, Mass., 1985.

[12] S. Chang, A closed hypersurface with constant scalar and mean curvatures in $S^{4}$ is isoparametric, Comm. Anal. Geom. 1, (1993), 71-100.

[13] J. Cheeger And M. Gromov, Bounds on the von Neumann dimension of $L^{2}$-cohomology and the GaussBonnet theorem for open manifolds, J. Differential Geom. 21 (1985), 1-34.

[14] B.-Y. CHEN, G-total curvature of immersed submanifolds, J. Differential Geom. 7 (1972), 371-391.

[15] S.-S. Chern, A simple intrinsic proof of the Gauss-Bonnet formula for closed Riemannian manifolds, Ann. of Math. (2) 45 (1944), 747-752.

[16] S.-S. CHERn, On the curvatura integra in a Riemannian manifold, Ann. of Math. (2) 46 (1945), 674-684.

[17] S.-S. Chern And R. K. LASHof, On the total curvature of immersed manifolds, Amer. J. Math. 79 (1957), 306-318, Part II: Michigan Math. J. 5 (1958), 5-12.

[18] S. Cohn-Vossen, Kürzeste Wege und Totalkrümmung auf Flächen, Compos. Math. 2 (1935), 69-133.

[19] F. Dillen AND W. KÜHNEL, Total curvature for open submanifolds of Euclidean spaces, Differential geometry (Sakado, 2001), 139-148, Josai Math. Monogr. 3, Josai Univ., Sakado, 2001.

[20] W. FENCHEL, On total curvatures of Riemannian manifolds: I, J. London Math. Soc. 15 (1940), 15-22.

[21] D. FERUS, Totale Absolutkrümmung in Differentialgeometrie und- topologie, Lecture Notes in Math. 66, Springer-Verlg, Berlin-New York, 1968.

[22] D. FERUS, On the type number of hypersurfaces in spaces of constant curvature, Math. Ann. 187 (1970), 310-316.

[23] D. FErus, Totally geodesic foliations, Math. Ann. 188 (1970), 313-316.

[24] F. FiAlA, Le problème isopérimètres sur les surfaces ouvertes à courbure positive, Comment. Math. Helv. 13 (1941), 293-346.

[25] A. FiALKOW, Hypersurfaces of a space of constant curvature, Ann. of Math. (2) 39 (1938), 762-785.

[26] R. FINN, On a class of conformal metrics, with application to differential geometry in the large, Comment. Math. Helv. 40 (1965), 1-30. 


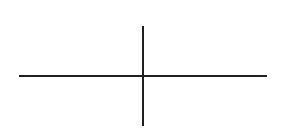

TOTAL CURVATURE OF COMPLETE SUBMANIFOLDS OF EUCLIDEAN SPACE

[27] M. VAn Gemmeren, Totale Krümmung und totale Absolutkrümmung von Untermannigfaltigkeiten des $\boldsymbol{R}^{m}$, Dissertation, Universität Stuttgart, Stuttgart, 1995.

[28] M. VAn Gemmeren, Total absolute curvature and tightness of noncompact manifolds, Trans. Amer. Math. Soc. 348 (1996), 2413-2426.

[29] D. H. Gottlieb, All the way with Gauss-Bonnet and the sociology of mathematics, Amer. Math. Monthly 103 (1996), 457-469.

[30] P. Hartman, Geodesic parallel coordinates in the large, Amer. J. Math. 86 (1964), 706-727.

[31] H. HopF, Über die Curvatura integra geschlossener Hyperflächen, Math. Ann. 95 (1926), 340-367.

[32] H. Hopf, Vektorfelder in n-dimensionalen Mannigfaltigkeiten, Math. Ann. 96 (1927), 225-250.

[33] H. Hopf, Über Flächen mit einer Relation zwischen den Hauptkrümmungen, Math. Nachr. 4 (1951), $232-249$.

[34] A. HubER, On subharmonic functions and differential geometry in the large, Comment. Math. Helv. 32 (1957), 13-72.

[35] A. HubER, Vollständige konforme Metriken und isolierte Singularitäten subharmonischer Funktionen, Comment. Math. Helv. 41 (1966/1967), 105-136.

[36] T. IshiHARA, The Euler characteristics and Weyl's curvature invariants of submanifolds in spheres, J. Math. Soc. Japan 39 (1987), 247-256.

[37] G. Ishikawa, M. Kimura And R. MiYaokA, Submanifolds with degenerate Gauss mappings in spheres, Lie groups, geometric structures and differential equations-one hundred years after Sophus Lie (Kyoto/Nara, 1999), 115-149, Adv. Stud. Pure Math. 37, Math. Soc. Japan, Tokyo, 2002.

[38] L. P. Jorge AND W. H. MeEKS III, The topology of complete minimal surfaces of finite total Gaussian curvature, Topology 22 (1983), 203-221.

[39] N. KLEInJohann And R. WALTER, Nonnegativity of the curvature operator and isotropy for isometric immersions, Math. Z. 181 (1982), 129-142.

[40] S. Kobayashi and K. NomizU, Foundations of Differential Geometry II, Interscience Tracts in Pure and Applied Mathematics 15 II, Interscience Publishers John Wiley \& Sons, Inc., New York-London-Sydney, 1969.

[41] P. Kohlmann, Uniqueness results for hypersurfaces in space forms, Habilitationsschrift Univ. Dortmund, 1995.

[42] Y. KUBO, A note on the scaling limit of a complete open surface, Tokyo J. Math. 18 (1995), 179-183.

[43] N. H. KUIPER, Der Satz von Gauss-Bonnet für Abbildungen in $E^{N}$ und damit verwandte Probleme, Jahresber. Deutsch. Math.-Verein. 69 (1967), 77-88.

[44] E. LEUZINGER, On the Gauss-Bonnet formula for locally symmetric spaces of noncompact type, Enseign. Math. (2) 42 (1996), 201-214.

[45] R. OsSERman, Global properties of minimal surfaces in $E^{3}$ and $E^{n}$, Ann. of Math. (2) 80 (1964), 340-364.

[46] M. PINL AND H. TRAPP, Stationäre Krümmungsdichten auf Hyperflächen des euklidischen $R_{n+1}$, Math. Ann. 176 (1968), 257-272.

[47] E. Portnoy, Toward a generalized Gauss-Bonnet formula for complete, open manifolds, Comment. Math. Helv. 46 (1971), 324-344.

[48] R. C. REILLY, Variational properties of functions of the mean curvatures for hypersurfaces in space forms, J. Differential Geom. 8 (1973), 465-477.

[49] S. Rosenberg, Gauss-Bonnet theorems for noncompact surfaces, Proc. Amer. Math. Soc. 86 (1982), 184 185.

[50] S. Rosenberg, On the Gauss-Bonnet theorem for complete manifolds, Trans. Amer. Math. Soc. 287 (1985), $745-753$.

[51] R. SACKS TEDER, On hypersurfaces with nonnegative sectional curvature, Amer. J. Math. 82 (1960), 609-630.

[52] L. A. SANTALó, Total curvatures of compact manifolds immersed in Euclidean space, Symposia Mathematica XIV (Convegni di Teoria Geometrica dell'Integrazione e Varietà Minimali, INDAM, Rome 1973), 363-390, Academic Press, London, 1974. 


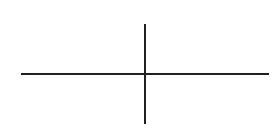

[53] V. A. Sharafutdinov, Relative Euler class and the Gauss-Bonnet theorem, Siberian Math. J. 14 (1974), 930-940.

[54] K. Shiонама, Busemann functions and total curvature, Invent. Math. 53 (1979), 281-297.

[55] K. Shiohama, The role of total curvature on complete noncompact Riemannian 2-manifolds, Illinois J. Math. 28 (1984), 597-620.

[56] K. Shiонамa, Total curvature and minimal areas of complete open surfaces, Proc. Amer. Math. Soc. 94 (1985), 310-316.

[57] K. Shiohama, T. Shioya And M. TANakA, Mass of rays on complete open surfaces, Pacific J. Math. 143 (1990), 349-358.

[58] T. Shioya, The ideal boundaries and global geometric properties of complete open surfaces, Nagoya Math. J. 120 (1990), 181-204.

[59] T. SHIOYA, The ideal boundaries of complete open surfaces, Tohoku Math. J. (2) 43 (1991), 37-59.

[60] T. Shioya, Geometry of total curvature, Acts de la Table Ronde de Géométrie Différentielle (Luminy, 1992), 561-600, Semin. Congr. 1, Soc. Math. France, Paris, (1996).

[61] J. J. STOKER, Über die Gestalt der positiv gekrümmten offenen Flächen im dreidimensionalen Raume, Compos. Math. 3 (1936), 55-88.

[62] E. TEUfEL, Anwendungen der differentialtopologischen Berechnung der totalen Krümmung und totalen Absolutkrümmung in der sphärischen Differentialgeometrie, Manuscripta Math. 32 (1980), 239-262.

[63] K. Voss, Einige differentialgeometrische Kongruenzsätze für geschlossene Flächen und Hyperflächen, Math. Ann. 131 (1956), 180-218.

[64] K. Voss, Variations of curvature integrals, Affine differential geometry (Oberwolfach, 1991), Results Math. 20 (1991), 789-796.

[65] R. WALTER, A generalized Allendoerffer-Weil formula and an inequality of the Cohn-Vossen type, J. Differential Geom. 10 (1975), 167-180.

[66] R. WALTER, Compact hypersurfaces with a constant higher mean curvature function, Math. Ann. 270 (1985), $125-145$.

[67] H. WeYL, On the volume of tubes, Amer. J. Math. 61 (1939), 461-472.

[68] B. WhiTe, Complete surfaces of finite total curvature, J. Differential Geom. 26 (1987), 315-326, Correction 28 (1988), 359-360.

[69] T. J. Willmore, Total curvature in Riemannian geometry, Ellis Horwood Series: Mathematics and its Applications, Ellis Horwood Ltd., Chicester, Halstead Press, New York, 1982.

[70] P. Wintgen, On total absolute curvature of nonclosed submanifolds, Ann. Global Anal. Geom. 2 (1984), $55-87$.

[71] H. Wu, A structure theorem for complete noncompact hypersurfaces of nonnegative curvature, Bull. Amer. Math. Soc. 77 (1971), 1070-1071.

[72] J.-W. Y IM, Convexity of the ideal boundary for complete open surfaces, Trans. Amer. Math. Soc. 347 (1995), $687-700$.

[73] K. Shiohama, T. Shioya And M. TAnaka, The geometry of total curvature on complete open surfaces, Cambridge Tracts in Math. 159, Cambridge Univ. Press, Cambridge, 2003.

[74] J. Milnor, On the immersion of $n$-manifolds in $(n+1)$-space, Comment. Math. Helv. 30 (1956), 275-284.

\section{Katholieke UniVersiteit LeUVEN}

DEPARTEMENT WISKUNDE

Celestiunenlaan 200B

B-3001 LEUVEN

BELGIUM

E-mail address: franki.dillen@wis.kuleuven.ac.be
FACHBEREICH MATHEMATIK

FAKULTÄT FÜR MATHEMATIK UND PHYSIK

UNIVERSITÄT STUTTGART

D-70550 STUTTGART

GERMANY

E-mail address: kuehnel@mathematik.uni-stuttgart.de 